\title{
Minimal self-joinings for nonsingular transformations
}

\author{
DANIEL J. RUDOLPH ${ }^{\dagger}$ AND CESAR E. SILVA \\ Department of Mathematics, University of Maryland, College Park, Maryland 20742, \\ USA; Department of Mathematics, Williams College, Williamstown, Massachusetts, \\ 01267, USA
}

(Received 13 October 1988)

\begin{abstract}
The notion of minimal self-joinings for conservative nonsingular actions is defined as a restriction on the nature of rational self-joinings. The need to consider rational joinings is demonstrated by showing that any two type $\mathrm{II}_{1}$ actions whose Cartesian product is ergodic have type $\mathrm{III}_{\lambda}$ nonsingular joinings. Lastly, actions of all Krieger types with minimal self-joinings are constructed. Hence these actions are prime and commute only with their powers.
\end{abstract}

\section{Introduction}

In [R], the notion of minimal self-joinings for a finite measure preserving transformation was introduced. Its original purpose was to provide a means of constructing examples of dynamical systems with certain prescribed behavior. This notion has grown and evolved since $[\mathbf{R}],[\mathbf{J R}]$.

Our purpose here is to generalize the notion of minimal self-joinings (in fact, just of 2-fold minimal self-joinings) to nonsingular conservative systems. Our basic motivation is to use this concept to construct nonsingular actions of various Krieger types which are prime and commute only with their powers. The original issue which began this work was a problem proposed by Choksi and Eigen concerning ergodic transformations on homogeneous measure algebras. This problem raised a number of questions in the Lebesgue case. In particular they asked whether an infinite measure preserving transformation could be prime. They also asked whether $T \times S$ could have Bernoulli factors, when $T$ is zero entropy mixing and $S$ is type III with no Bernoulli factors. Our work answers the former in the affirmative; the latter question remains open.

Aaronson and Nadkarni in [AN] construct an example of an infinite measure preserving transformation with no nontrivial $\sigma$-finite factors. Their example is a group rotation and does have non $\sigma$-finite factors. Maharam has asked whether

\footnotetext{
$†$ Research partially supported by NSF Grant DM 88-02593.

$¥$ Research done while visiting the Department of Mathematics, University of Maryland, College Park. Partially supported by NSF Grant DMS 86-01619.
} 
there exist infinite measure preserving transformations with no nontrivial $0-\infty$ sub- $\sigma$-algebras. Our examples all have ergodic Cartesian squares and no nontrivial factor algebras at all. (We let factor algebra mean factor sub- $\sigma$-algebra, and identify factor algebras $(\bmod 0)$; the trivial algebras are the full algebra and $\{\varnothing, X\}$.)

The notion of a joining of two measures is by now well understood. In the measure preserving case, it is natural to require the joining to also be invariant for the product action. In the nonsingular case, it would seem natural to ask that the joining simply be nonsingular for the product. This is inadequate. As we shall see, it does not reduce to the measure preserving definition. In fact, we show that any two finite measure preserving actions whose direct product is ergodic have nonsingular joinings of type III $_{\lambda}$ for any $0<\lambda<1$. As with much of our work, this argument leaves many open questions (cf. Question A). They are more appropriately stated through the text.

In order to obtain a useful definition of minimal self-joinings we must restrict ourselves to a class of joinings we call rational (cf. $\S 2$ ). The joinings critical for our needs, i.e. relatively independent joinings over factor algebras and joinings supported on a single graph, are rational. Moreover any rational joining of type $\mathrm{II}_{1}$ 's is of type $\mathrm{II}_{1}$. Thus we do obtain the finite measure preserving theory as a special case.

Minimal self-joinings is defined as a condition on an action with respect to a certain collection of equivalent measures. In the finite measure preserving case there is just one measure, the invariant measure, to consider, but in the nonsingular case no particular measure is distinguished.

The definition also differs from the measure preserving case in that we restrict all rational joinings, not just the ergodic ones. The reason is that an ergodic component of a nonsingular joining need not be a joining of measures equivalent to the original [A]. This is true in the measure preserving case though, so once more our definition reduces to the standard one there.

We investigate the structure of rational joinings when they are of minimal type. Our arguments suggest many open questions.

Lastly, we construct actions of each Krieger type having minimal self-joinings with respect to a sufficiently large class of measures to guarantee they are prime and commute only with their powers.

\section{Preliminaries}

\subsection{Basic definitions}

Let $(X, \mathscr{B}, \mu)$ be a probability measure space. A nonsingular transformation (or nonsingular automorphism) $T$ is a bijective bimeasurable map of $X$ such that $T(A)$ is null if and only if $A$ is null. We let $\mu \circ T$ denote the measure $\mu \circ T(A)=\mu(T(A))$. The Radon-Nikodym derivative of $T^{i}, i \in \mathbb{Z}$, is defined to be

$$
\omega_{i}(T ; x)=\omega_{i}(x)=\frac{d \mu \circ T^{i}}{d \mu}(x) .
$$

After discarding an invariant null set we may assume that the functions $\omega_{i}$ are 
positive and finite everywhere. We have,

$$
\begin{gathered}
\omega_{i+j}(x)=\omega_{i}(x) \omega_{j}\left(T^{i} x\right) \quad \mu \text {-a.e. and } \\
\int f d \mu=\int f \circ T^{i} \omega_{i} d \mu \quad \text { for all } f \in L^{1}(\mu)
\end{gathered}
$$

A dynamical system $(X, \mathscr{B}, \mu, T)$ consists of a standard space $(X, \mathscr{B})$, a Borel probability measure $\mu$, and a nonsingular automorphism $T:(X, \mathscr{B}, \mu) \rightarrow(X, \mathscr{B}, \mu)$. In the sequel, all measure spaces are standard probability spaces. If the measure $\mu$ is completed with respect to null sets then $(X, \mathscr{B}, \mu)$ is a Lebesgue probability space, and we may complete the measures and make use of the properties of Lebesgue spaces without explicit references. We may sometimes simply write $(X, \mu, T)$ or $(T, \mu)$ instead of $(X, \mathscr{B}, \mu, T)$.

A nonsingular factor map is a map $\varphi:(X, \mathscr{B}, \mu, T) \rightarrow(W, \mathscr{D}, \rho, U)$ such that:

(i) $\varphi^{-1}(\mathscr{D}) \subset \mathscr{B}$,

(ii) $\mu \circ \varphi^{-1} \approx \rho$, i.e., they are equivalent measures, and

(iii) $\varphi \circ T=U \circ \varphi$.

In this case we say that $(W, \mathscr{D}, \rho, U)$ is a (nonsingular) factor of $(X, \mathscr{B}, \mu, T)$. If $\varphi$ is a nonsingular factor map then $\varphi^{-1}(\mathscr{D})$ is a $T$-invariant sub- $\sigma$-algebra of $\mathscr{B}$. It can be shown that all nonsingular factors can be identified in this way (up to nonsingular isomorphism); we will use both representations interchangeably. We note that the measure $\rho$ can be replaced by an equivalent measure, namely $\mu{ }^{\circ} \varphi^{-1}$, so that $\varphi$ becomes measure preserving. If no transformations are involved, we do not require condition (iii) of the definition but still call $\varphi$ a nonsingular factor map.

A transformation $T$ is conservative if $\mu\left(A \backslash \bigcup_{i \geqslant 1} T^{-i} A\right)=0$ for all $A \in \mathscr{B}$. This is equivalent to $\sum_{i=0}^{\infty} \omega_{i}=\infty \mu$-a.e. [M1]. It is easy to see that an ergodic nonsingular automorphism is not conservative if and only if it is the shift map on $\mathbf{Z}$. We shall use the following version of the ergodic theorem.

HuREWICZ ERGOdic THEOREM 1.1.1. ([H].) Let $(X, \mathscr{B}, \mu, T)$ be a dynamical system. If $T$ is conservative and $f$ is integrable then

$$
\lim _{n \rightarrow \infty} \frac{\sum_{i=0}^{n-1} f \circ T^{i} \omega_{i}}{\sum_{i=0}^{n-1} \omega_{i}}=\mathbb{E}_{\mu}[f \mid \mathscr{I}],
$$

where $\mathbb{E}_{\mu}$ denotes the usual conditional expectation function, and $\mathscr{I}$ the sub- $\sigma$ algebra of invariant sets.

\subsection{The ratio set}

We shall need a few basic facts from Krieger's classification of nonsingular automorphisms - we refer to $[\mathbf{K r}]$ or $[\mathbf{H O}],[\mathbf{S}],[\mathbf{W}]$ for further details and proofs of the statements below.

Let $T$ be a conservative ergodic nonsingular automorphism of $(X, \mathscr{B}, \mu)$. The ratio set $r(T)$ of $T$ is defined to be

$$
\begin{aligned}
& r(T)=\left\{t \in \mathbb{R}^{+} \cup\{0\}: \forall A \in \mathscr{B}, \mu(A)>0, \forall \varepsilon>0,\right. \\
& \exists n \neq 0 \text { and } B \subset A, \mu(B)>0, \quad \text { such that } \\
&\left.T^{n}(B) \subset A \text { and }\left|\omega_{n}(x)-t\right|<\varepsilon \forall x \in B\right\},
\end{aligned}
$$


$r(T)$ depends only on the measure class of $\mu$, and $r(T) \backslash\{0\}$ is a closed multiplicative subgroup of $\mathbb{R}^{+}$. Furthermore, $r(T)$ is an invariant of orbit equivalence.

$r(T)=\{1\}$ if and only if $(T, \mu)$ admits an equivalent $\sigma$-finite invariant measure; in this case we say $T$ is type $I I$. If the equivalent invariant measure is finite, $T$ is type $I I_{1}$ and if it is infinite it is type $I_{\infty}$. If $0 \in r(T)$ then $(T, \mu)$ admits no equivalent $\sigma$-finite invariant measure and is said to be type III. Further, $T$ is type $I I I_{\lambda}, 0<\lambda<1$, if $r(T)=\left\{\lambda^{n}: n \in \mathbb{Z}\right\} \cup\{0\}, T$ is type $I I_{0}$ if $r(T)=\{0,1\}$, and $T$ is type $I I I_{1}$ if $r(T)=\mathbb{R}^{+} \cup\{0\}$. Transformations in different Krieger types are not orbit equivalent, hence not isomorphic.

It is clear that a factor of a type $\mathrm{II}_{1}$ transformation must be type $\mathrm{II}_{1}$, but a type $\mathrm{II}_{\infty}$ may have type III factors.

\subsection{Disintegrations}

Let $\varphi:(X, \mathscr{B}, \mu) \rightarrow(W, \mathscr{D}, \rho)$ be a nonsingular factor map. Then there exists (see e.g. [M: $\S 2])$ a disintegration of $(X, \mathscr{B}, \mu)$ with respect to $\varphi$. That is, for each $w \in W$ there exists a measure $\mu_{w}$ on $\mathscr{B} \cap \varphi^{-1}(w)$ such that:

(i) The real valued function

$$
w \rightarrow \mu_{w}\left(A \cap \varphi^{-1}(w)\right)
$$

is $\mathscr{D}$-measurable,

(ii) $\mu=\int \mu_{w} d \rho(w)$.

Furthermore, condition (ii) determines the disintegration almost uniquely. We may denote the disintegration by $\left\{\mu_{\varphi(x)}\right\}$. It follows from (ii) that for any integrable function $f$,

$$
\int f d \mu=\iint f d \mu_{w} d \rho(w) .
$$

Let $\mathscr{D}$ be a factor of $(X, \mathscr{B}, \mu, T)$, and let $\mu_{\mathscr{P}}, T_{\mathscr{X}}$, and $\varphi$ denote the factor measure, transformation and map. We have the following useful formula.

$$
\frac{d \mu \circ T}{d \mu}(x)=\frac{d \mu_{\mathscr{P}} \circ T_{\mathscr{A}}}{d \mu_{\mathscr{I}}}(\varphi(x)) \frac{d \mu_{\varphi(T(x))} \circ T}{d \mu_{\varphi(x)}}(x) \quad \mu \text {-a.e., }
$$

where $\left\{\mu_{\varphi(x)}\right\}$ denotes the disintegration of $\mu$ with respect to $\mathscr{D}$. This equation follows from the relation,

$$
\int \chi_{A} \frac{d \mu \circ T}{d \mu} d \mu=\int \chi_{A} \frac{d \mu_{\mathscr{P}} \circ T}{d \mu_{\mathscr{P}}}(x) \frac{d \mu_{\varphi(T(x))^{\circ} T}}{d \mu_{\varphi(x)}} d \mu, \quad \text { for all } A \in \mathscr{B},
$$

which can be proved using (1.1.1) and (1.3.1).

We refer to $[\mathbf{S : ~} \$ 6]$ for a proof of the following theorem.

ERGodic DECOMPOSITION THEOREM 1.3.1. Let $(X, \mathscr{B}, \mu, T)$ be a dynamical system. There exists a standard space $(Z, \mathscr{C})$ and a nonsingular factor map $\theta:(X, \mathscr{B}, \mu) \rightarrow$ $(Z, \mathscr{C}, \zeta), \zeta=\mu \circ \theta^{-1}$, such that $(X, \mathscr{B}, \mu)$ disintegrates over $\theta$, i.e., there is a disintegration $\left\{\nu_{z}\right\}$, and furthermore

(i) for all $z \in Z, \nu_{z}$ is nonsingular and ergodic for $T$,

(ii) if $\mu$ is invariant under $T$ then so is every $\nu_{z}$, 
(iii) if $\mu$ is type II then so is every $\nu_{z}$,

(iv) $\nu_{z}\left(\theta^{-1}\{z\}\right)=1$ for all $z \in Z$. In particular, $\nu_{z_{1}} \perp \nu_{z_{2}}$ whenever $z_{1} \neq z_{2}$,

(v) $\theta^{-1}(\mathscr{C}) \subset \mathscr{I}$ and $\theta^{-1}(\mathscr{C})=\mathscr{I}(\bmod \mu)$.

(vi) (Uniqueness) If $\theta^{\prime}:(X, \mathscr{B}, \mu) \rightarrow\left(Z^{\prime}, \mathscr{C}^{\prime}, \zeta^{\prime}\right)$ is another nonsingular factor map and $\left\{\nu_{z}^{\prime}\right\}$ is a disintegration of $(X, \mathscr{B}, \mu)$ with respect to $\theta^{\prime}$ satisfying (i)-(v) then there is a nonsingular isomorphism $\varphi:(Z, \mathscr{C}, \zeta) \rightarrow\left(Z^{\prime}, \mathscr{C}^{\prime}, \zeta^{\prime}\right)$ such that $\nu_{\varphi(z)}^{\prime} \approx \nu_{z}$ for a.e. $z \in Z$.

(vii) When $X$ is nonatomic, $T$ is conservative if and only if $\nu_{z}$ is not the shift on $\mathbf{Z}$.

\subsection{Joinings}

Let $\left(X_{1}, \mathscr{B}_{1}, \mu_{1}\right),\left(X_{2}, \mathscr{B}_{2}, \mu_{2}\right)$ be two standard spaces. A joining of $\mu_{1}$ and $\mu_{2}$ is a probability measure $\hat{\mu}$ on $\mathscr{B}_{1} \times \mathscr{B}_{2}$ such that

$$
\begin{array}{ll}
\hat{\mu}\left(A \times X_{2}\right)=\mu_{1}(A) & \text { for all } A \in \mathscr{B}_{1}, \text { and } \\
\hat{\mu}\left(X_{1} \times B\right)=\mu_{2}(B) & \text { for all } B \in \mathscr{B}_{2} .
\end{array}
$$

Suppose $\left(X_{1}, \mathscr{B}_{1}, \mu_{1}\right),\left(X_{2}, \mathscr{B}_{2}, \mu_{2}\right)$ have a common factor $(W, \mathscr{D}, \rho)$, i.e., there are nonsingular factor maps

$$
\begin{aligned}
& \varphi_{1}:\left(X_{1}, \mathscr{B}_{1}, \mu_{1}\right) \rightarrow(W, \mathscr{D}, \rho), \text { and } \\
& \varphi_{2}:\left(X_{2}, \mathscr{B}_{2}, \mu_{2}\right) \rightarrow(W, \mathscr{D}, \rho) .
\end{aligned}
$$

Let $\left\{\mu_{1, w}\right\},\left\{\mu_{2, w}\right\}$ be the disintegrations of $\mu_{1}$ and $\mu_{2}$ over $\varphi_{1}$ and $\varphi_{2}$, respectively. The relatively independent joining $\hat{\mu}$ of $\mu_{1}$ and $\mu_{2}$ over $(W, \rho)$ (or over the factor algebra $\mathscr{D}$ ) is defined by

$$
\hat{\mu}=\int \mu_{1, w} \times \mu_{2, w} d \rho(w) .
$$

It is easy to check that $\hat{\mu}$ is a joining (cf. [F2], [JR] for an in-depth treatment and basic properties of joinings that will be used here).

A nonsingular joining of two dynamical systems $\left(X_{1}, \mathscr{B}_{1}, \mu_{1}, T_{1}\right)$ and $\left(X_{2}, \mathscr{B}_{2}, \mu_{2}, T_{2}\right)$ is a joining $\hat{\mu}$ of $\mu_{1}$ and $\mu_{2}$ such that $T_{1} \times T_{2}$ is nonsingular for $\hat{\mu}$. If $(W, \mathscr{D}, \rho, U)$ is a factor of $\left(X_{1}, \mathscr{B}_{1}, \mu_{1}, T_{1}\right)$ and $\left(X_{2}, \mathscr{B}_{2}, \mu_{2}, T_{2}\right)$, then the relatively independent joining of $\mu_{1}$ and $\mu_{2} \operatorname{over}(W, \rho)$ is a nonsingular joining of $\left(X_{1}, \mu_{1}, T_{1}\right)$ and $\left(X_{2}, \mu_{2}, T_{2}\right)$. Clearly, product measure is also a nonsingular joining.

We will be mainly interested in self-joinings, that is nonsingular joinings $\hat{\mu}$ of $\left(X, \mu_{1}, T\right)$ with $\left(X, \mu_{2}, T\right)$, i.e. joinings or nonsingular joinings over the same space with perhaps different but equivalent measures. A non-singular self-joining $\hat{\mu}$ is said to be of graph form if $\hat{\mu}$ is supported on some (single) graph

$$
G_{j}=\left\{\left(x, T^{j}(x)\right\}\right. \text {. }
$$

A nonsingular joining totally supported on a union of graphs $G_{j}$ is called a graphic joining.

1.5. Notation

$$
\begin{aligned}
& \omega_{i}^{1}(x)=\frac{d \mu_{1} \circ T_{1}^{i}}{-d \mu_{1}}(x) \quad \text { (When } i=1 \text { we drop the subscript.) } \\
& \omega_{i}^{2}(y)=\frac{d \mu_{2} \circ T_{2}^{i}}{d \mu_{2}}(y)
\end{aligned}
$$




$$
\hat{\omega}_{i}(x, y)=\frac{d \hat{\mu} \circ\left(T_{1} \times T_{2}\right)^{i}}{d \hat{\mu}}(x, y) .
$$

We let $\left\{\nu_{z}\right\}$ stand for the ergodic decomposition of the nonsingular joining $\hat{\mu} . \nu_{z}^{1}$ and $\nu_{z}^{2}$ denote the $X_{1}$ and $X_{2}$-marginals of $\nu_{z} \cdot \hat{\mu}_{x}$ and $\hat{\mu}^{y}$ denote the vertical and horizontal fibre measures of $\hat{\mu}$, respectively.

\section{Rational joinings}

TheOREM 2.1. Let $\left(X_{1}, \mathscr{B}_{1}, \mu_{1}, T_{1}\right)$ and $\left(X_{2}, \mathscr{B}_{2}, \mu_{2}, T_{2}\right)$ be two finite measure preserving systems such that $T_{1} \times T_{2}$ is ergodic. For every $\lambda, 0<\lambda<1$, there exists $a$ nonsingular joining $\hat{\mu}$ of $\mu_{1}$ and $\mu_{2}$ such that $\left(T_{1} \times T_{2}, \hat{\mu}\right)$ is conservative ergodic type $\mathrm{III}_{\lambda}$.

The proof proceeds in a series of lemmas. What we will do is construct, on a series of Rohlin towers, modifications of product measure. Each successive modification will project on its marginals to $\mu_{1}$ and $\mu_{2}$, and in fact the RadonNikodym cocycle will be a coboundary. The weak limit of these modifications will be our desired measure.

By a rectangle we mean a set of the form $A \times B, A \in \mathscr{B}_{1}, B \in \mathscr{B}_{2}$. Let $A \in \mathscr{B}_{1}, B \in \mathscr{B}_{2}$ and $0<\lambda<1$. We will describe a function $w_{A, B}: A \times B \rightarrow\left\{\lambda^{i}\right\}_{i \in \mathbb{Z}}$ with the properties:

(i) $\left\{(x, y): w_{A, B}(x, y)=\lambda^{i}\right\}$ is a finite union of rectangles,

(ii) $\mu_{1} \times \mu_{2}\left(\left\{(x, y): w_{A, B}(x, y)=\lambda\right\}=\frac{1}{2} \mu_{1}(A) \mu_{2}(B)\right.$, and

(iii) for all $x_{0} \in A, y_{0} \in B$,

$$
\int_{B} w_{A, B}\left(x_{0}, y\right) d \mu_{2}(y)=\mu_{2}(B) \text { and } \int_{A} w_{A, B}\left(x, y_{0}\right) d \mu_{1}(x)=\mu_{1}(A) .
$$

Select $i(j) \rightarrow_{j} \infty$ so that

$$
a(j)=\frac{1-\lambda}{2\left(\lambda^{i(j)}-1\right)}<2^{-j-1} .
$$

Inductively select $n(j) \in \mathbb{Z}^{+}$so that

$$
\frac{1}{2}-\left(2^{-J}\right) \leq \sum_{j=1}^{J} n(j) a(j)<\frac{1}{2}-\left(2^{-J-1}\right) .
$$

This gives

$$
\sum_{j=1}^{\infty} n(j) a(j)=\frac{1}{2}
$$

Pick $A_{0} \subset A$ with $\mu_{1}\left(A_{0}\right)=\frac{1}{2} \mu_{1}(A)$ and $B_{0} \subset B$ with $\mu_{2}\left(B_{0}\right)=\frac{1}{2} \mu_{2}(B)$. Partition $A_{0}$ into subsets $A_{j, k}, j=1, \ldots, \infty, k=1, \ldots, n(j)$ with $\mu_{1}\left(A_{j, k}\right)=a(j) \mu_{1}(A)$. This is possible since $\sum n(j) a(j) \mu_{1}(A)=\mu_{1}(A) / 2$.

Similarly partition $A_{0}^{c}, B_{0}$ and $B_{0}^{c}$ into $A_{j, k}^{\prime}, B_{j, k}$, and $B_{j, k}^{\prime}$ with $\mu_{1}\left(A_{j, k}^{\prime}\right)=a(j) \mu_{1}(A)$ and $\mu_{2}\left(B_{j, k}\right)=\mu_{2}\left(B_{j, k}^{\prime}\right)=a(j) \mu_{2}(B)$.

Define $w_{A, B}$ to be:

(a) $\lambda$, for $(x, y) \in A_{0} \times B_{0}$ or $A_{0}^{c} \times B_{0}^{c}$,

(b) $\lambda^{i(j)}$ for $(x, y) \in A_{j, k} \times B_{j, k}^{\prime}$ or $A_{j, k}^{\prime} \times B_{j, k}$, and

(c) 1 otherwise. 
Conditions (a) and (b) are clear. For (c) we compute the case for $x_{0} \in A_{0}$. The rest are symmetric.

For some unique $j, k, x_{0} \in A_{j, k}$,

$$
\begin{aligned}
\int_{B} w_{A, B}\left(x_{0}, y\right) d \mu_{2}(y)= & \int_{B_{0}} w_{A, B}\left(x_{0}, y\right) d \mu_{2}(y) \\
& +\int_{B_{i, k}^{\prime}} w_{A, B}\left(x_{0}, y\right) d \mu_{2}(y)+\int_{B_{0}^{c} \backslash B_{j, k}^{\prime}} w_{A, B}\left(x_{0}, y\right) d \mu_{2}(y) \\
= & \lambda \mu_{2}\left(B_{0}\right)+\lambda^{-i(j)} \mu_{2}\left(B_{j, k}^{\prime}\right)+\mu_{2}\left(B_{0}^{c} \backslash B_{j, k}^{\prime}\right) \\
= & \mu_{2}\left(B_{0}\right)+\frac{\lambda-1}{2} \mu_{2}(B)+\mu_{2}\left(B_{j, k}^{\prime}\right) \\
& +\left(\lambda^{-i(j)}-1\right) \mu_{2}\left(B_{j, k}^{\prime}\right)+\mu_{2}\left(B_{0}^{c} \backslash B_{j, k}^{\prime}\right) \\
= & \mu_{2}(B)+\left(\frac{\lambda-1}{2}+\left(\lambda^{-i(j)}-1\right) a(j)\right) \mu_{2}(B) \\
= & \mu_{2}(B) \quad \text { since }\left(\lambda^{-i(j)}-1\right) a(j)=(1-\lambda) / 2 .
\end{aligned}
$$

We now show how to use $w_{A, B}$ to modify a joining $\hat{\mu}$ of $\mu_{1}$ and $\mu_{2}$. Suppose we are given such a joining. Suppose further we are given a partition $H$ of $X_{1} \times X_{2}$. Suppose we are given a rectangle $R=A_{0} \times B_{0}$ satisfying:

(i) $\hat{\mu}$ restricted to $R$ is of the form $c_{0}\left(\mu_{1} \times \mu_{2}\right)$, i.e. a constant multiple of product measure.

(ii) For $i=0,1, \ldots, 3 N-1$, for $(x, y) \in R, d \hat{\mu} \circ\left(T_{1}^{i} \times T_{2}^{i}\right) / d \hat{\mu}(x, y)=d_{i}$ is a constant,

(iii) For $i=0,1, \ldots, 3 N-1, T_{1}^{i} \times T_{2}^{i}(R)=A_{i} \times B_{i}$ is contained in a single element of $H$, and the sets $A_{i} \times B_{i}$ are disjoint.

We call the sequence of sets $A_{i} \times B_{i}$ a $(\hat{\mu}, H)$-pure column of length $3 N$.

On such a pure column we can modify $\hat{\mu}$ as follows. On the level sets $T_{1}^{i} \times T_{2}^{i}(R)=A_{i} \times B_{i}, N \leq i<2 N$, define

$$
\hat{\mu}^{\prime}\left(D \cap\left(A_{i} \times B_{i}\right)\right)=\int_{A_{i} \times B_{i}} w_{A_{0} \times B_{0}}\left(T_{1}^{-i}(x), T_{2}^{-i}(y)\right) \chi_{D}(x, y) d \hat{\mu} .
$$

Leave $\hat{\mu}$ unchanged elsewhere.

LEMMA 2.1. For $\hat{\mu}^{\prime}$ defined as above, $\hat{\mu}^{\prime}$ is a joining of $\mu_{1}$ and $\mu_{2}$ and for any set $D \in H, \hat{\mu}^{\prime}(D)=\hat{\mu}(D)$.

Proof. Notice that $\hat{\mu}^{\prime}$ is equivalent to $\hat{\mu}$ and

$$
\frac{d \hat{\mu}^{\prime}}{d \hat{\mu}}(x, y)=w_{A_{i} \times B_{0}}\left(T_{1}^{-i}(x), T_{2}^{-i}(y)\right) \text { for }(x, y) \in A_{i} \times B_{i} \text {, and } 1 \text { elsewhere. }
$$

Thus for any $D \in \mathscr{B}_{1}$,

$$
\hat{\mu}^{\prime}\left(D \times X_{2}\right)=\hat{\mu}\left(\left(\left(D \times X_{2}\right) \cap\left(\bigcup_{i=N}^{2 N-1} A_{i} \times B_{i}\right)^{c}\right)+\sum_{i=N}^{2 N-1} \hat{\mu}^{\prime}\left(\left(D \cap A_{i}\right) \times B_{i}\right) .\right.
$$


Now $\hat{\mu}$ on $A_{i} \times B_{i}$ is $c_{0} d_{i}\left(\mu_{1} \times \mu_{2}\right)$, so

$$
\begin{aligned}
\hat{\mu}^{\prime}\left(\left(D \cap A_{i}\right) \times B_{i}\right) & =c_{0} d_{i} \int_{D \cap A_{i}}\left(\int_{B_{0}} w_{A_{0} \times B_{0}}(x, y) d \mu_{2}\right) d \mu_{1} \\
& =c_{0} d_{i} \mu_{2}\left(B_{i}\right) \mu_{1}\left(D \cap A_{i}\right) \\
& =\hat{\mu}\left(\left(D \cap A_{i}\right) \times B_{i}\right) .
\end{aligned}
$$

Thus $\hat{\mu}^{\prime}\left(D \times X_{2}\right)=\hat{\mu}\left(D \times X_{2}\right)=\mu_{1}(D)$. Similarly, the other marginal is still $\mu_{2}$.

If $D \in H$, then any $A_{i} \times B_{i}$ that intersects $D$ nontrivially is actually contained in $D$. A computation identical to that given above now tells us $\hat{\mu}^{\prime}(D)=\hat{\mu}(D)$.

Suppose we had some countable collection $R^{1}, R^{2}, \ldots$, of bases for $(\hat{\mu}, H)$-pure columns of height $3 N$, all of which were disjoint. We could simultaneously modify all of the columns to obtain a new joining of $\mu_{1}$ and $\mu_{2}$. We call the new joining $\hat{\mu}^{\prime}$ obtained this way the $\lambda, N$-modification of $\hat{\mu}$ on the columns over $R^{1}, R^{2}, \ldots$ Let $\left\{P_{i}\right\}$ and $\left\{Q_{i}\right\}$ be refining and generating sequences of partitions of $X_{1}$ and $X_{2}$, respectively. We will define a sequence of joinings $\hat{\mu}_{i}$. We start with $\hat{\mu}_{0}=\mu_{1} \times \mu_{2}$. Inductively we want:

(i) $\hat{\mu}_{i}$ is equivalent to $\hat{\mu}_{0}$ and $d \hat{\mu}_{i} / d \hat{\mu}_{0}$ takes on values $\left\{\lambda^{j}\right\}$ only and its level sets are disjoint unions of rectangles.

(ii) $\hat{\mu}_{i+1}$ is a $\lambda, N_{i}$-modification of $\hat{\mu}_{i}$ on some collection of $\left(\hat{\mu}_{i}, p_{i} \times Q_{i}\right)$-pure columns. $N_{i+1} \geq 2^{10(i+1)^{2}} N_{i}$ and the columns cover a set of $\hat{\mu}_{0}$ measure at least 0.9 .

(iii) Because of (i) and (ii) above, there is a countable partition $E_{i}$ of $X_{1} \times X_{2}$ into rectangles so that on any element of $E_{i}, d \hat{\mu}_{i} / d \hat{\mu}_{0}$ is a constant. Let $d_{1}, \ldots, d_{t}$ be a finite collection of atoms of $E_{i}$ with $\hat{\mu}_{i}\left(\bigcup_{j=1}^{t} d_{j}\right)>1-2^{-10 i}$. Let $B_{u}$ be the atoms of $P_{i+1} \times Q_{i+1}$ and $A=d_{k} \cap B_{u}$, for some $k, u$. The value $N_{i+1}$ is so large that on a set of $\hat{\mu}_{i}$ measure at least $1-2^{-10 i}$, for $0 \leq n \leq N_{i+1}$, for all $D$ as above,

$$
\sum_{j=-n}^{N_{i+1}-n-1} \chi_{A}\left(T_{1}^{j} \times T_{2}^{j}(x, y)\right) \geq 0.9 N_{i+1} \hat{\mu}_{0}(A) .
$$

LEMMA 2.2. For $\left(X_{1}, \mathscr{B}_{1}, \mu_{1}, T_{1}\right)$ and $\left(X_{2}, \mathscr{B}_{2}, \mu_{2}, T_{2}\right)$ finite measure preserving processes with $T_{1} \times T_{2} \mu_{1} \times \mu_{2}$-ergodic, there is a sequence of joinings satisfying (i), (ii) and (iii) above.

Proof. We start with $\hat{\mu}_{0}=\mu_{1} \times \mu_{2}$. Suppose we have constructed $\hat{\mu}_{i}$. We need first to select $N_{i+1}$. We require $N_{i+1} \geq 2^{10(i+1)^{2}} N_{i}$ and having selected $d_{1}, \ldots, d_{1}$ atoms of $E_{i}$ with $\hat{\mu}_{i}\left(\bigcup_{j=1}^{\prime} d_{j}\right)>1-2^{-10 i}$, the Birkhoff theorem gives us a further lower bound for $N_{i+1}$ so that for $0 \leq n<N_{i+1}$, for all $A$ as in (iii),

$$
\sum_{j=-n}^{N_{i+1}-n-1} \chi_{A}\left(T_{1}^{j} \times T_{2}^{j}(x, y)\right) \geq 0.9 N_{i+1} \hat{\mu}_{0}(A)
$$

on a set of $(x, y)$ of $\hat{\mu}_{0}$ measure at least $1-2^{-10 n}$. Using this $N_{i+1}$ we must construct the pure columns. In $\left(X_{1}, T_{1}\right)$ select a Rohlin base $F_{i+1}$ for a tower of height $3 N_{i+1}$ and error 0.01 . 
Now $F_{i+1} \times X_{2}$ is a rectangular Rohlin base for $T_{1} \times T_{2}$. The span

$$
\bigvee_{j=0}^{3 N_{i+1}^{-1}}\left(T_{1} \times T_{2}\right)^{-j}\left(E_{i} \vee P_{i+1} \times Q_{i+1}\right)
$$

partitions $F_{i+1} \times X_{2}$ into rectangular bases for $\left(\hat{\mu}_{i}, P_{i+1} \times Q_{i+1}\right)$-pure columns of height $3 N_{i+1}$. Let $\hat{\mu}_{i+1}$ be the $\lambda, N_{i+1}$-modification of $\hat{\mu}$ on the columns over these bases.

COROLlaRy 2.3. The joinings $\hat{\mu}_{i}$ converge weakly to a joining $\hat{\mu}$ of $\mu_{1}$ and $\mu_{2}$. Furthermore, $T_{1} \times T_{2}$ is nonsingular for $\hat{\mu}$ and the ratio set of $T_{1} \times T_{2}$ with respect to $\hat{\mu}$ is contained in $\left\{\lambda^{i}\right\} \cup\{0\}$.

Proof. Since $\hat{\mu}_{i+1}$ is a $\lambda, N_{i+1}$-modification of $\hat{\mu}_{i}$ on $\left(\hat{\mu}_{i}, P_{i+1} \times Q_{i+1}\right)$-pure columns, for any set

$$
D \in P_{i+1} \times Q_{i+1}, \hat{\mu}_{i+1}(D)=\hat{\mu}_{i}(D) .
$$

As the $P_{i} \times Q_{i}$ refine, for any $j \geq i, \hat{\mu}_{j}(D)=\hat{\mu}_{i}(D)$. Thus $\hat{\mu}$ is well defined and finitely additive on the dense algebra (not $\sigma$-algebra) of sets generated by $\bigcup_{i} P_{i} \times Q_{i}$.

Of course $\hat{\mu}$ projects to $\mu_{1}$ and $\mu_{2}$ on its marginals. This then implies that $\hat{\mu}$ extends to a joining measure on the $\sigma$-algebra $\mathscr{B}_{1} \times \mathscr{B}_{2}$, since $\hat{\mu}$ satisfies the Kolmogorov extension criteria.

Let $L_{i}=T^{N_{i}-1}\left(F_{i}\right) \cup T^{2 N_{i}-1}\left(F_{i}\right)$. For $(x, y) \notin L_{i} \times X_{2}$ we easily compute

$$
\frac{d \hat{\mu}_{i} \circ\left(T_{1} \times T_{2}\right)}{d \hat{\mu}_{i}}(x, y)=\frac{d \hat{\mu}_{i-1} \circ\left(T_{1} \times T_{2}\right)}{d \hat{\mu}_{i-1}}(x, y)
$$

as it is on level $T^{N_{1}}\left(F_{i}\right) \times X_{2}$ of the Rohlin tower that the $w_{A, B}$ are first introduced, and on the $T^{2 N_{i}}\left(F_{i}\right) \times X_{2}$ they are removed.

Now $\mu_{1}\left(L_{i}\right)<2^{-10 i+1}$. Thus for $\hat{\mu}_{0}$ and $\hat{\mu}$-a.e. $(x, y),(x, y)$ belongs to only finitely many $L_{i}$, and hence as $i$ increases

$$
\frac{d \hat{\mu}_{i} \circ\left(T_{1} \times T_{2}\right)}{d \hat{\mu}_{i}}(x, y)
$$

ceases to vary on an ever larger subset of $X_{1} \times X_{2}$. Since $\hat{\mu}_{i} \rightarrow \hat{\mu}$ weakly, this limit must be

$$
\frac{d \hat{\mu} \circ\left(T_{1} \times T_{2}\right)}{d \hat{\mu}}(x, y)
$$

and must lie in $\left\{\lambda_{i}\right\}_{i \in \mathbb{Z}}$. We conclude $T_{1} \times T_{2}$ is nonsingular and its ratio set with respect to $\hat{\mu}$ is contained in $\left\{\lambda^{i}\right\} \cup\{0\}$.

To complete Theorem 2.1 we only need show that $\lambda$ itself is in the ratio set.

LeMmA 2.4. For $\hat{\mu}_{i}$ as constructed above, for any set $C \in P_{i} \times Q_{i}$ there is a subset $C^{\prime} \subset C$ with

$$
\hat{\mu}_{i-1}\left(C^{\prime}\right)>0.4 \hat{\mu}_{i-1}(C)-2^{-10(i-1)}
$$


$C^{\prime}$ is contained in the first $N_{i}$ levels of the Rohlin tower over $F_{i} \times X_{2}$. For $(x, y) \in C^{\prime}$ there is an $e(x, y), 1 \leq e(x, y) \leq 2 N^{\prime}$, so that

$$
\left(T_{1} \times T_{2}\right)^{e(x, y)}(x, y): C^{\prime} \rightarrow C \text { is } 1-1
$$

mapping $C^{\prime}$ into the middle $N_{i}$ levels of the Rohlin tower over $F_{i} \times X_{2}$. Lastly,

$$
\frac{d \hat{\mu}_{i} \circ\left(T_{1} \times T_{2}\right)^{e(x, y)}}{d \hat{\mu}_{i}}(x, y)=\lambda .
$$

Proof. Let $d_{1}, \ldots, d_{i}$ be atoms of $E_{i-1}$ with $\hat{\mu}_{i-1}\left(\bigcup_{j=1}^{t} d_{j}\right)>1-2^{-10(i-1)}$. Let $C_{j}=C \cap d_{j}$ and $\hat{\mu}_{i-1}\left(\bigcup_{j=1}^{t} C_{j}\right) \geq \hat{\mu}_{i-1}(C)-2^{-10(i-1)}$. Let $G \subset F_{i} \times X_{2}$ consist of those $(x, y)$ such that for all $j$,

$$
\begin{aligned}
& \sum_{k=0}^{N_{i}-1} \chi_{C_{i}}\left(T_{1}^{k} \times T_{2}^{k}(x, y)\right) \geq 0.9 N_{i} \hat{\mu}_{0}\left(C_{j}\right) \quad \text { and } \\
& \sum_{k=N_{i}}^{2 N_{i}-1} \chi_{C_{i}}\left(T_{1}^{k} \times T_{2}^{k}(x, y)\right) \geq 0.9 N_{i} \hat{\mu}_{0}\left(C_{j}\right) .
\end{aligned}
$$

If $(x, y) \notin G$, then either

(a) for all $0 \leq k<N_{i}, T_{1}^{k} \times T_{2}^{k}(x, y)$ does not satisfy (iii) of Lemma 2.2, or

(b) for all $N_{i} \leq k<2 N_{i}, T_{1}^{k} \times T_{2}^{k}(x, y)$ does not satisfy (iii) of Lemma 2.2.

Thus $\hat{\mu}_{0}(G)>\mu\left(F_{i}\right)\left(1-2 \cdot 2^{-10 i}\right)$. On any of the $\left(\hat{\mu}_{i-1}, P_{i+1} \times Q_{i+1}\right)$-pure columns in the Rohlin tower over $F_{i} \times X_{2}, \chi_{C}$ is a constant on levels. Hence $G$ is a union of bases of such pure columns. Let $A \times B$ be a base for a pure column in $G$. For each $C_{j}$ select subsequences

$$
0 \leq k_{i}^{j}<k_{2}^{j}<\cdots<k_{l(j)}^{j}<N_{i} \text { and } N_{i} \leq k_{1}^{j^{\prime}}<k_{2}^{j^{\prime}}<\cdots<k_{l(j)}^{j^{\prime}}<2 N_{i}
$$

with

$$
\begin{aligned}
& \left(T_{1} \times T_{2}\right)^{k_{\prime \prime}^{\prime}}(A \times B) \subset C_{j}, \\
& \left(T_{1} \times T_{2}\right)^{k_{\prime \prime}^{\prime}}(A \times B) \subset C_{j}, \quad \text { and } \\
& l(j)>0.9 N_{i} \hat{\mu}_{0}\left(C_{j}\right) .
\end{aligned}
$$

Consider the set $A_{0} \times B_{0} \cup A_{0}^{c} \times B_{0}^{c}$ on which $w_{A_{0}, B_{0}}(x, y)=\lambda$. Let

$$
C^{\prime}=\bigcup_{A \times B \subset G} \bigcup_{u=1}^{(j)}\left(T_{1} \times T_{2}\right)^{k^{\prime}(}\left(A_{0} \times B_{0} \cup A_{0}^{c} \times B_{0}^{c}\right) \subset C .
$$

Now $d \hat{\mu}_{i-1} / d \hat{\mu}_{0}$ is a constant on $d_{j}$ and hence $C_{j}$. Call its value $\delta_{j}$.

$$
\begin{aligned}
\hat{\mu}_{i-1}\left(C^{\prime}\right) & =\sum_{j} l(j) \delta_{j} \hat{\mu}_{0}(G) / 2 \\
& \geq 0.9 N_{i}\left(1-2 \cdot 2^{-10 i}\right) \mu_{1}\left(F_{i}\right) \sum_{j} \delta_{j} \hat{\mu}_{0}\left(C_{j}\right) / 2 \\
& \geq \frac{0.81}{2}\left(1-2 \cdot 2^{-10 i}\right) \sum_{j} \hat{\mu}_{i-1}\left(C_{j}\right) \\
& =\frac{0.81}{2}\left(1-2 \cdot 2^{-10 i}\right) \hat{\mu}_{i-1}\left(C \cap \bigcup_{j=1}^{1} d_{j}\right) \\
& \geq 0.4 \hat{\mu}_{i-1}\left(C_{j}\right)-2^{-10(i-1)} .
\end{aligned}
$$


For $A \times B$ in $G$, for $(x, y) \in\left(T_{1} \times T_{2}\right)^{k_{1}^{j}}\left(A_{0} \times B_{0} \cup A_{0}^{c} \times B_{0}^{c}\right)$, define $e(x, y)=k_{u}^{j^{\prime}}-k_{u}^{j}$. Now $\left(T_{1} \times T_{2}\right)^{e(x, y)}(x, y): C^{\prime} \cap C_{j} \rightarrow C_{j}$ is $1-1$ and since $d \hat{\mu}_{i-1} / d \hat{\mu}_{0}$ is a constant on $C_{j}$,

$$
\frac{d \hat{\mu}_{i-1} \circ\left(T_{1} \times T_{2}\right)^{e(x, y)}}{d \hat{\mu}_{i-1}}(x, y)=1 .
$$

But in constructing $\hat{\mu}_{i}$, for $(x, y) \in C^{\prime} \cap C_{j}$ we obtain

$$
\begin{aligned}
& \frac{d \hat{\mu}_{i} \circ\left(T_{1} \times T_{2}\right)^{e(x, y)}}{d \hat{\mu}_{i}}(x, y) \\
& =w_{A_{0}, B_{0}}\left(\left(T_{1} \times T_{2}\right)^{-k_{1 \prime}^{j}}(x, y)\right) \frac{d \hat{\mu}_{i-1} \circ\left(T_{1} \times T_{2}\right)^{e(x, y)}}{d \hat{\mu}_{i-1}}(x, y)=\lambda .
\end{aligned}
$$

COROLlARY 2.5. $\lambda$ is in the ratio set of $T_{1} \times T_{2}$ acting on $\hat{\mu}$.

Proof. To begin, consider the set

$$
L_{i}=\bigcup_{j=N_{i}-3 N_{i-1}}^{N_{i}+3 N_{i-1}-1} T^{j}\left(F_{i}\right) \cup \bigcup_{j=2 N_{i}-3 N_{i-1}}^{2 N_{i}+3 N_{i-1}-1} T^{j}\left(F_{i}\right)
$$

If $(x, y) \notin L_{i} \times X_{2}$ and $0 \leq k<3 N_{i-1}$ then

$$
\frac{d \hat{\mu}_{i} \circ\left(T_{1} \times T_{2}\right)^{k}}{d \hat{\mu}_{i}}(x, y)=\frac{d \hat{\mu}_{i-1} \circ\left(T_{1} \times T_{2}\right)^{k}}{d \hat{\mu}_{i-1}}(x, y)
$$

as along the piece of orbit $(x, y),\left(T_{1}(x), T_{2}(y)\right), \ldots,\left(T_{1}^{k}(x), T_{2}^{k}(y)\right)$ we never cross from using to not using some $w_{A, B}$. Now

$$
\mu\left(L_{i}\right) \leq \frac{12 N_{i-1}}{N_{i}} \leq \frac{12}{2^{10(i)^{2}}}
$$

Thus a point $(x, y)$ lies in at most finitely many $L_{i}$. For any set $C, \hat{\mu}(C)>0$, select $i$ large enough and $\bar{C} \in P_{i} \times Q_{i}$ so that $\hat{\mu}(\bar{C} \Delta C) \leq(\lambda / 100) \hat{\mu}(C)$ and also by the previous lemma there is a $\bar{C}^{\prime} \subset \bar{C}, \bar{C}^{\prime}$ in the first third of the tower over $F_{i} \times X_{2}$,

$$
\hat{\mu}_{i-1}\left(\bar{C}^{\prime}\right)>0.3 \hat{\mu}_{i-1}(\bar{C})=0.3 \hat{\mu}(\bar{C})
$$

Furthermore, $i$ is so large that

$$
\mu\left(\bigcup_{j=i}^{\infty} L_{j}\right)<0.1 \hat{\mu}(\vec{C})=0.1 \hat{\mu}_{i}(\vec{C})
$$

For $(x, y) \in \bar{C}^{\prime}$ we know

$$
\frac{d \hat{\mu}_{i} \circ\left(T_{1} \times T_{2}\right)^{e(x, y)}}{d \hat{\mu}_{i}}(x, y)=\lambda
$$

If furthermore $(x, y) \notin \bigcup_{j=i}^{\infty} L_{j}$, then

$$
\frac{d \hat{\mu} \circ\left(T_{1} \times T_{2}\right)^{e(x, y)}}{d \hat{\mu}}(x, y)=\frac{d \hat{\mu}_{i} \circ\left(T_{1} \times T_{2}\right)^{e(x, y)}}{d \hat{\mu}_{i}}(x, y)=\lambda .
$$

Now $\hat{\mu}\left(\bar{C}^{\prime} \backslash \bigcup_{j=i}^{\infty} L_{j}\right) \geq 0.2 \hat{\mu}(\bar{C})$.

Letting

$$
C^{\prime}=\left\{(x, y) \in \bar{C}^{\prime} ¥ \bigcup_{j=i}^{\infty} L_{j}:(x, y) \in C \text { and }\left(T_{1} \times T_{2}\right)^{e(x, y)} \in C\right\},
$$




$$
\begin{gathered}
\qquad \begin{array}{c}
\hat{\mu}\left(C^{\prime}\right) \geq 0.2 \hat{\mu}(\bar{C})-\frac{\lambda}{100} \hat{\mu}(C)-\frac{1}{100} \hat{\mu}(C) \\
\geq(0.2-0.02) \hat{\mu}(C)>0 . \\
\text { For }(x, y) \in C^{\prime},\left(T_{1} \times T_{2}\right)^{e(x, y)}(x, y) \in C \text { and } \\
\frac{d \hat{\mu} \circ\left(T_{1} \times T_{2}\right)^{e(x, y)}}{d \hat{\mu}}(x, y)=\lambda,
\end{array}
\end{gathered}
$$

and so $\lambda$ is in the ratio set.

Theorem 2.1 shows that weakly mixing finite measure preserving transformations are not strongly simple [A], thus answering a question of Aaronson's [A].

Question A. What Krieger types are obtainable as nonsingular joinings of two actions of particular types? Can type $\mathrm{HI}_{\lambda}$ of Theorem 2.1 be replaced with other Krieger types? Type $\mathrm{III}_{1}$ as a joining of $\mathrm{II}_{1}$ 's is an easy modification of our argument. Can $\mathrm{II}_{\infty}$ be obtained? Beyond the obvious restriction that a $\mathrm{II}_{1}$ joining implies $\mathrm{II}_{1}$ marginals, are other restrictions forced? How many distinct isomorphism classes of type $\mathrm{III}_{\lambda}$ can be obtained? If the original systems are of other Krieger types, what types of joinings can exist?

Definition. Let $\hat{\mu}$ be a nonsingular joining of $\left(X_{1}, \mathscr{B}_{1}, \mu_{1}, T_{1}\right)$ and $\left(X_{2}, \mathscr{B}_{2}, \mu_{2}, T_{2}\right)$. We say that $\hat{\mu}$ is a rational joining of $\mu_{1}$ and $\mu_{2}$ if

(i) for $\mu_{1}$-a.e. $x$,

$$
\frac{\hat{\omega}\left(x, y_{1}\right)}{\hat{\omega}\left(x, y_{2}\right)}=\frac{\omega^{2}\left(y_{1}\right)}{\omega^{2}\left(y_{2}\right)}, \quad \hat{\mu}_{x} \text {-a.e. }
$$

(ii) for $\mu_{2}$-a.e. $y$,

$$
\frac{\hat{\omega}\left(x_{1}, y\right)}{\hat{\omega}\left(x_{2}, y\right)}=\frac{\omega^{1}\left(x_{1}\right)}{\omega^{1}\left(x_{2}\right)}, \quad \hat{\mu}^{y} \text {-a.e. }
$$

We may regard $\omega^{1}(x)$ and $\omega^{2}(y)$ as functions on $X_{1} \times X_{2}$ defined $\hat{\mu}$-a.e. Define

$$
c(x, y)=\frac{\hat{\omega}(x, y)}{\omega^{1}(x) \omega^{2}(y)} .
$$

$c(x, y)$ is defined and finite positive $\hat{\mu}$-a.e. By integrating the fiber measures in (i) and (ii) above, it is clear the equalities in (i) and (ii) actually hold $\hat{\mu}$-a.e. Thus $c(x, y)$ is $\hat{\mu}$-a.e. constant on vertical and horizontal fibers. The converse is also true, $\hat{\mu}$ is rational if $c(x, y)$ is $\hat{\mu}$-a.e. constant on vertical and horizontal fibers.

It follows that a rational joining of finite measure preserving systems is finite measure preserving, since $c(x, y)=\hat{\omega}(x, y)$ and $\int \hat{\omega}(x, y) d \hat{\mu}_{x}=1$, so $\hat{\omega}=1 \hat{\mu}$-a.e.

We note that the ergodic components of a (conservative) rational joining need not be nonsingular joinings (see [A: p. 260]).

PROPOSITION 2.6. The following nonsingular joinings are rational.

(i) Product measure.

(ii) Joinings of graph form.

(iii) Relatively independent joinings over factor algebras. 
Proof. It is clear that product measure and joinings of graph form are rational. Now let $\hat{\mu}$ be the relatively independent joining over the factor algebra $\mathscr{D}$. By (1.3.2),

$$
\frac{d \hat{\mu}^{\circ}(T \times T)}{d \hat{\mu}}(x, y)=\frac{d \hat{\mu}_{\mathscr{Q}} \circ(T \times T)}{d \hat{\mu}_{\mathscr{Q}}}(\hat{\varphi}(x, y)) \frac{d \hat{\mu}_{\hat{\varphi}(T \times T)(x, y)}{ }^{\circ}(T \times T)}{d \hat{\mu}_{\hat{\varphi}(x, y)}}(x, y) \hat{\mu} \text {-a.e. }
$$

By the properties of the relatively independent joining,

$$
\frac{d \hat{\mu}_{\mathscr{D}}^{\circ}(T \times T)}{d \hat{\mu}_{\mathscr{D}}}(\hat{\varphi}(x, y))=\frac{d \mu_{\mathscr{T}} \circ T}{d \mu_{\mathscr{P}}}(\varphi(x)) \hat{\mu} \text {-a.e.. }
$$

So

$$
\begin{aligned}
\frac{d \hat{\mu} \circ(T \times T)}{d \hat{\mu}}(x, y) & =\frac{d \mu_{\mathscr{Q}} \circ T_{\mathscr{Q}}}{d \mu_{\mathscr{P}}}(\varphi(x)) \frac{d \mu_{\varphi(T x)} \circ T}{d \mu_{\varphi(x)}}(x) \frac{d \mu_{\varphi(T y)} \circ T}{d \mu_{\varphi(y)}}(y) \\
& =\frac{d \mu \circ T}{d \mu}(x) \frac{d \mu \circ T}{d \mu}(y) \frac{1}{\frac{d \mu_{\mathscr{D}} \circ T_{\mathscr{Q}}}{d \mu_{\mathscr{D}}}(\varphi(y))} .
\end{aligned}
$$

Thus $c(x, y)$, for fixed $y$, is independent of $x$. The symmetric fact is, of course, also true.

\section{Minimal self-joinings}

\subsection{Basic properties}

Definition. A dynamical system $(X, \mathscr{B}, \mu, T)$ has minimal self-joinings over $\mathfrak{M}$, where $\mathfrak{M}$ is a class of probability measures equivalent to $\mu$, if for every $\mu_{1}, \mu_{2} \in \mathfrak{M}$, for every rational joining $\hat{\mu}$ of $\mu_{1}$ and $\mu_{2}$, for a.e. ergodic component $\nu_{z}$ of $\hat{\mu}$ we have:

(i) $\nu_{z}=\nu_{z}^{1} \times \nu_{z}^{2}$, i.e. is the product of its marginals, or

(ii) $\nu_{z}$ is of graph form.

For finite measure preserving transformations, minimal self-joinings over $\{\mu\}$ is the same as the usual (2-fold) minimal self-joinings.

Minimal self-joinings restricts the nature of the ergodic components of a rational joining. We now investigate the consequences of this restriction. We start with a useful technical result (Proposition 3.1.5) which is essential to a number of later results.

LemMA 3.1.1. Let $\left(X_{1}, \mathscr{B}_{1}, \mu_{1}, T_{1}\right)$ and $\left(X_{2}, \mathscr{B}_{2}, \mu_{2}, T_{2}\right)$ be two dynamical systems and $\nu$ be a measure on $X_{1} \times X_{2}$ nonsingular and conservative ergodic for $T_{1} \times T_{2}$. If $\nu$ is nonsingular for $T_{1} \times I$, and

$$
\frac{d \nu \circ T_{1} \times I}{d \nu}(x, y)=\frac{d \nu \circ T_{1}}{d \nu^{1}}(x) \nu \text {-a.e., }
$$

then $\nu=\nu^{1} \times \nu^{2}$, the product of its marginals. 
Proof. We have

$$
\begin{aligned}
\nu(A \times B) & =\int \mathbb{E}\left[\chi_{A \times B} \mid \Phi_{T_{1} \times I}\right] d \nu \\
& =\int \lim _{n \rightarrow \infty} \frac{\sum_{i=0}^{n-1} \chi_{A \times B} \circ\left(T_{1} \times I\right)^{i}(x, y) \frac{d \nu \circ\left(T_{1} \times I\right)^{i}}{d \nu}(x, y)}{\sum_{i=0}^{n-1} \frac{d \nu \circ\left(T_{1} \times I\right)^{i}}{d \nu}(x, y)} d \nu \\
& =\int \lim _{n \rightarrow \infty} \frac{\sum_{i=0}^{n-1} \chi_{A} \circ T_{1}^{i}(x) \chi_{B}(y) \frac{d \nu^{1} \circ T_{1}^{i}}{d \nu^{1}}(x)}{\sum_{i=0}^{n-1} \frac{d \nu^{1} \circ T_{1}^{i}}{d \nu^{1}}(x)} d \nu \\
& =\int \nu^{1}(A) \chi_{B}(y) d \nu=\nu^{1}(A) \nu^{2}(B) .
\end{aligned}
$$

LEMMA 3.1.2. Let $\hat{\mu}$ be a rational joining of $\left(X_{1}, \mathscr{B}_{1}, \mu_{1}, T_{1}\right)$ and $\left(X_{2}, \mathscr{B}_{2}, \mu_{2}, T_{2}\right)$. Then for a.e. ergodic component $\nu_{z}$ of $\hat{\mu}$, if $\nu_{z}$ is nonsingular for $T_{1} \times I$, then

$$
\frac{d \nu_{z} \circ\left(T_{1} \times I\right)}{d \nu_{z}}=\frac{d \nu_{z}^{1} \circ T_{1}}{d \nu_{z}^{1}} \quad \nu_{z} \text {-a.e. }
$$

Proof. Let $A=\left\{z: \nu_{z}\right.$ is $T_{1} \times I$ nonsingular $\}$. Since $\hat{\mu}$ is rational,

$$
\hat{\omega}(x, y)=\omega^{1}(x) \omega^{2}(y) c(x, y) \hat{\mu} \text {-a.e., }
$$

where $c$ is $\hat{\mu}$-a.e. constant on horizontal and vertical fibres. So

$$
c(x, y)=c\left(T_{1} x, y\right)=c\left(T_{1} x, T_{2} y\right) \nu_{z} \text {-a.e }
$$

Therefore $c(x, y)=c$, a constant, $\nu_{z}$-a.e., and

$$
\frac{d \nu_{z} \circ\left(T_{1} \times T_{2}\right)}{d \nu_{z}}(x, y)=\frac{d \mu_{1} \circ T_{1}}{d \mu_{1}}(x) \frac{d \mu_{2} \circ T_{2}}{d \mu_{2}}(y) c \quad \nu_{z} \text {-a.e. }
$$

Using this equality and the chain rule on $\left(T_{1} \times T_{2}\right) \circ\left(T_{1} \times I\right)=\left(T_{1} \times I\right) \circ\left(T_{1} \times T_{2}\right)$ we obtain

$$
\begin{aligned}
& \frac{d \mu_{1} \circ T_{1}}{d \mu_{1}}(x) \frac{d \mu_{2} \circ T_{2}}{d \mu_{2}}(y) c \frac{d \nu_{z} \circ\left(T_{1} \times I\right)}{d \nu_{z}}\left(T_{1} x, T_{2} y\right) \\
& \quad=\frac{d \nu_{z} \circ\left(T_{1} \times I\right)}{d \nu_{z}}(x, y) \frac{d \mu_{1} \circ T_{1}}{d \mu_{1}}\left(T_{1} x\right) \frac{d \mu_{2} \circ T_{2}}{d \mu_{2}}(y) c \quad \nu_{z} \text {-a.e. }
\end{aligned}
$$

So

$$
\frac{\frac{d \nu_{z} \circ\left(T_{1} \times I\right)}{d \nu_{z}}(x, y)}{\frac{d \mu_{1} \circ T_{1}}{d \mu_{1}}(x)}=\frac{\frac{d \nu_{z} \circ\left(T_{1} \times I\right)}{d \nu_{z}}\left(T_{1} x, T_{2} y\right)}{\frac{d \mu_{1} \circ T_{1}}{d \mu_{1}}\left(T_{1} x\right)} \quad \nu_{z} \text {-a.e. }
$$


Put

$$
g(x, y)=\frac{\frac{d \nu_{z} \circ\left(T_{1} \times I\right)}{d \nu_{z}}(x, y)}{\frac{d \mu_{1} \circ T_{1}}{d \mu_{1}}(x)} .
$$

Then $g$ is well-defined $\nu_{z}$-a.e. and $g=g \circ\left(T_{1} \times T_{2}\right) \nu_{z}$-a.e. Thus $g=K(z) \nu$-a.e. and

$$
\frac{d \nu_{z} \circ\left(T_{1} \times I\right)}{d \nu_{z}}(x, y)=K(z) \frac{d \mu_{1} \circ T_{1}}{d \mu_{1}}(x) \nu_{z} \text {-a.e. }
$$

Now let $\left.\nu_{z}\right|_{x},\left.\nu_{z}\right|_{T_{1} x}$ denote the fibre measures of $\nu_{z}$ at $x$ and $T_{1} x$, respectively. Then by (1.3.2) we have,

$$
\frac{d \nu_{z} \circ\left(T_{1} \times I\right)}{d \nu_{z}}(x, y)=\frac{d \nu_{z}^{1} \circ T_{1}}{d \nu_{z}^{1}}(x) \frac{\left.d \nu_{z}\right|_{T_{2} x}}{\left.d \nu_{z}\right|_{x}}(y) .
$$

Thus,

$$
\frac{\left.d \nu_{z}\right|_{T_{1} x}}{\left.d \nu_{z}\right|_{x}}(y)=K(z) \frac{\frac{d \mu_{1} \circ T_{1}}{d \mu_{1}}(x)}{\frac{d \nu_{z}^{1} \circ T_{1}}{d \nu_{z}^{1}}(x)}
$$

Integrating with respect to $\left.d \nu_{z}\right|_{x}(y)$ gives,

$$
\frac{d \nu_{z}^{1} \circ T_{1}}{d \nu_{z}^{1}}(x)=K(z) \frac{d \mu_{1} \circ T_{1}}{d \mu_{1}}(x) \nu_{z} \text {-a.e. }
$$

LEMMA 3.1.3. Suppose $\left(X_{1}, T_{1}, \mu_{1}\right)$ and $\left(X_{2}, T_{2}, \mu_{2}\right)$ are both ergodic and $\hat{\mu}$ is $a$ nonsingular joining. Further suppose

$$
\hat{\mu}=\sum_{i=1}^{\infty} \alpha_{i} \hat{\mu}_{i}, \quad \text { where } \alpha_{i} \geq 0, \sum \alpha_{i}=1,
$$

and each $\hat{\mu}_{i}$ is $\left(T_{1} \times T_{2}\right)$-nonsingular. If $\hat{\mu}_{i}^{1}$ and $\hat{\mu}_{i}^{2}$ are the marginals of $\hat{\mu}_{i}$, then for each $i$ with $\alpha_{i}>0, \hat{\mu}_{i}^{j}$ are equivalent to $\mu_{j}, j=1,2$.

Proof. If $\hat{\mu}_{i}^{1}(A)>0$ then $\mu_{1}(A)=\hat{\mu}\left(A \times X_{2}\right)>\alpha_{i} \hat{\mu}_{i}^{1}(A)>0$. On the other hand, if $\mu_{1}(A)>0$ then $\mu_{1}\left(\bigcup_{k=-\infty}^{\infty} T_{1}^{k}(A)\right)=1$ so $\hat{\mu}_{i}^{\prime}\left(\bigcup_{k=-\infty}^{\infty} T_{1}^{k}(A)\right)=1$ and since $T_{1}$ is $\hat{\mu}_{i}^{1}$-nonsingular, $\hat{\mu}_{i}^{1}(A)>0$.

LEMMA 3.1.4. Let $\hat{\mu}$ be a rational joining of $\left(X_{1}, \mathscr{B}_{1}, \mu_{1}, T_{1}\right)$ and $\left(X_{2}, \mathscr{B}_{2}, \mu_{2}, T_{2}\right)$. Then for a.e. conservative ergodic component $\nu_{z}$ of $\hat{\mu}$, if $\nu_{z}$ is nonsingular for $T_{1} \times I$,

$$
\frac{d \mu_{1} \circ T_{1}}{d \mu_{1}}=\frac{d \nu_{z}^{1} \circ T_{1}}{d \nu_{z}^{\prime}} \nu_{z}^{1} \text {-a.e. }
$$

Proof. Let $A=\left\{z: \nu_{z}\right.$ is $T_{1} \times I$ nonsingular $\}$. We show that in equation (3.1.1), $K=1$ for $\zeta$-a.e. $z \in A$. Now given $B \subset Z, \zeta(B)>0$ (cf. Theorem 1.3.1), define

$$
\nu_{B}=\frac{1}{\zeta(B)} \int_{B} \nu_{z} d \zeta(z) .
$$


Let $\nu_{B}^{1}$ and $\nu_{B}^{2}$ denote the $X_{1}$ - and $X_{2}$-marginals of $\nu_{B}$, respectively. Then

$$
\hat{\mu}=\zeta(B) \nu_{B}+(1-\zeta(B)) \nu_{B^{i}} .
$$

It follows from Lemma 3.1.3 that $\mu_{1} \approx \nu_{B}^{1}$. Now suppose $K(z) \geq k>1$ for $z \in B \subset A$. Then

$$
\frac{d \nu_{B} \circ\left(T_{1} \times I\right)}{d \nu_{B}}(x, y) \geq k \frac{d \mu_{1} \circ T_{1}}{d \mu_{1}}(x)
$$

so

$$
\frac{d \nu_{B}^{1} \circ T_{1}}{d \nu_{B}^{1}} \geq k \frac{d \mu_{1} \circ T_{1}}{d \mu_{1}}(x), \quad \text { or } \quad \frac{d \nu_{B}^{1} \circ T_{1}}{d \nu_{B}^{1}} \frac{d \mu_{1}}{d \mu \circ T_{1}}(x) \geq k .
$$

But since $\mu_{1} \approx \nu_{B}^{\prime}$, we would have a coboundary $>1$, contradicting the conservativity of $T_{1}$, so $K(z) \leq 1 \zeta$-a.e. on $A$ and similarly $K(z) \geq 1 \zeta$-a.e. on $A$.

Proposition 3.1.5. Let $\left(X_{1}, \mathscr{B}_{1}, \mu_{1}, T_{1}\right)$ and $\left(X_{2}, \mathscr{B}_{2}, \mu_{2}, T_{2}\right)$ be two conservative ergodic dynamical systems. Let $\hat{\mu}$ be a rational joining of $\mu_{1}$ and $\mu_{2}$. Then for a.e. conservative ergodic component $\nu_{z}$ of $\hat{\mu}$, if $\nu_{z}$ is nonsingular for $T_{1} \times I$, and hence for $I \times T_{2}$, then $\nu_{z}=\mu_{1} \times \mu_{2}$.

Proof. From the previous lemmas we have that $\nu_{z}=\nu_{z}^{1} \times \nu_{z}^{2}$, and so $\nu_{z}$ is $I \times T_{2}$ nonsingular. Let $A=\left\{z: \nu_{z}\right.$ is $T_{1} \times I$ and $I \times T_{2}$ nonsingular $\}$. Write

$$
\omega^{z}(x)=d \nu_{z}^{1} \circ T_{1} / d \nu_{z}^{1} \text {. }
$$

Then $\omega_{i}^{z}(x)=\omega_{i}^{1}(x) \nu_{z}$-a.e. Let $\left\{f_{j}\right\}$ be a dense family of functions in $\left(X_{1}, \mathscr{B}_{1}\right)$ so that the Hurewicz ergodic theorem holds after removing a null set. Then for all $x \in X_{1}$, and all $j$,

$$
\begin{aligned}
& \lim _{n \rightarrow \infty} \frac{\sum_{i=0}^{n-1} f_{j}\left(T_{1}^{i}(x)\right) \omega_{i}^{1}(x)}{\sum_{i=0}^{n-1} \omega_{i}^{1}(x)}=\int f_{j} d \mu_{1}, \text { and } \\
& \lim _{n \rightarrow \infty} \frac{\sum_{i=0}^{n-1} f_{j}\left(T_{1}^{i}(x)\right) \omega_{i}^{z}(x)}{\sum_{i=0}^{n-1} \omega_{i}^{z}(x)}=\int f_{j} d \nu_{z}^{1} .
\end{aligned}
$$

Thus $\int f_{j} d \mu_{1}=\int f_{j} d \nu_{z}^{1}$. Since this holds for a dense family of functions then $\mu_{1}=\nu_{z}^{1}$, and similarly $\mu_{2}=\nu_{z}^{2}$.

Let $\hat{\mu}$ be a nonsingular joining of $\left(T_{1}, \mu_{1}\right)$ and $\left(T_{2}, \mu_{2}\right)$. We say that an ergodic component $\nu_{z}$ of $\hat{\mu}$ is of product form if $\nu_{z}=\nu_{z}^{1} \times \nu_{z}^{2}$, the product of its marginals.

Corollary 3.1.6. Suppose $\left(T_{1}, \mu_{1}\right)$ and $\left(T_{2}, \mu_{2}\right)$ are conservative ergodic and $\hat{\mu}$ is a rational joining. Then a.e. ergodic component $\nu_{z}$ of product form is $\mu_{1} \times \mu_{2}$ and must be conservative.

Proof. First we show a.e. $\nu_{z}$ of product form is conservative. If not then $\nu_{z}$ is the shift map on the integers. Thus either $\left(T_{1}, \nu_{z}^{1}\right)$ or $\left(T_{2}, \nu_{z}^{2}\right)$ must be the identity on a one point space, and the other is the shift map on the integers as this is the only way the shift can be written as a product. Let $A_{i}=\left\{z \in Z:\left(T_{i}, \nu_{z}^{i}\right)\right.$ is the shift map on the integers\}. If $\zeta\left(A_{1} \cup A_{2}\right)=0$ we are done. But if $\zeta\left(A_{i}\right)>0$, then

$$
\bar{\nu}=\frac{1}{\zeta\left(A_{i}\right)} \int_{A_{i}} \nu_{z}^{i} d \zeta(z)
$$


is absolutely continuous with respect to $\mu_{i}$ by Lemma 3.1 .4 but $\left(T_{i}, \bar{\nu}\right)$ is not conservative.

Now for $\nu_{2}$ conservative and of product form, $\nu_{z}$ is $T_{1} \times I$ nonsingular and by Proposition 3.1.5 we are finished.

LEMMA 3.1.7. Suppose $\hat{\mu}$ is a rational joining of $\left(X_{1}, T_{1}, \mu_{1}\right)$ and $\left(X_{2}, T_{2}, \mu_{2}\right)$ and $\hat{\mu}=\alpha \nu_{1}+(1-\alpha) \nu_{2}, \alpha \neq 0,1$, and the $\nu_{i}$ are nonsingular for $\left(T_{1} \times T_{2}\right)$, but are mutually singular. If $\nu_{1}$ is a rational joining of $\mu_{1}$ and $\mu_{2}$, then $\nu_{2}$ is also.

Proof. Since both $\hat{\mu}$ and $\nu_{1}$ have $\mu_{1}$ and $\mu_{2}$ are marginals, so does $\nu_{2}$. As $\nu_{1}$ and $\nu_{2}$ are mutually singular, they are supported on disjoint invariant sets and

$$
\frac{d \hat{\mu} \circ\left(T_{1} \times T_{2}\right)}{d \hat{\mu}}(x, y)=\frac{d \nu_{2} \circ\left(T_{1} \times T_{2}\right)}{d \nu_{2}}(x, y)
$$

for $(x, y) \in \operatorname{supp}\left(\nu_{2}\right)$. Hence $\nu_{2}$ is rational.

Corollary 3.1.8. Suppose $\hat{\mu}$ is a rational joining of $\left(X_{1}, T_{1}, \mu_{1}\right)$ and $\left(X_{2}, T_{2}, \mu_{2}\right)$ almost all of whose ergodic components are of product form or of graph form. Then

$$
\hat{\mu}=\alpha\left(\mu_{1} \times \mu_{2}\right)+(1-\alpha) \hat{\mu}_{G},
$$

where $\hat{\mu}_{G}$ is a rational joining of $\mu_{1}$ and $\mu_{2}$ almost all of whose ergodic components are of graph form.

Proof. By Corollary 3.1.6 a.e. ergodic component $\nu_{z}$ of product form is $\mu_{1} \times \mu_{2}$. Letting $\alpha$ be the measure of this set, it is in fact a single ergodic component. The complementary set supports $\hat{\mu}_{G}$. That $\hat{\mu}_{G}$ is a rational joining follows from our previous lemma.

Proposition 3.1.9. If $\left(T_{1}, \mu_{1}\right)$ and $\left(T_{2}, \mu_{2}\right)$ are conservative ergodic and $\hat{\mu}$ is $a$ rational joining almost all of whose ergodic components are of product form or graph form, then $\hat{\mu}$ is conservative.

Proof. From Corollaries 3.1.6 and 3.1.8 we can assume $\hat{\mu}=\hat{\mu}_{G}$ is of graph form. Thus $\hat{\mu}=\sum_{i=1}^{\infty} \alpha_{i} \hat{\mu}_{i}$, where $\hat{\mu}_{i}$ is supported on $G_{i}$. By Lemma 3.1.3, if $\alpha_{i}>0$, then $\hat{\mu}_{i}^{j}$ is absolutely continuous with respect to $\mu_{j}$. Thus $\left(T_{1} \times T_{2}, \hat{\mu}_{i}^{j}\right)$ is nonsingularly conjugate to $\left(T_{j}, \mu_{j}\right)$ and is conservative.

LEMMA 3.1.10. If $\hat{\mu}$ is a graphic rational joining supported on a single graph $G_{j}$ then $\mu_{1}=\mu_{2} \circ T^{j}$.

Proof. The projections $\pi_{1}:\left(x, T^{j}(x)\right) \rightarrow x$ and $\pi_{2}:\left(x, T^{j}(x)\right) \rightarrow T^{j}(x)$ satisfy $\mu_{1} \circ \pi_{1}=\hat{\mu}=\mu_{2} \circ \pi_{2}$. As they are 1-1 their inverses are nonsingular and $\mu_{1}=\mu_{2} \circ \pi_{2} \circ \pi_{1}^{-1}=\mu_{2} \circ T^{j}$.

THEOREM 3.1.11. Suppose $(X, \mathscr{B}, \mu, T)$ is conservative totally ergodic and has minimal self-joinings over $\mathfrak{M}$. If $S \in C(T)(=$ the commutant of $T)$ and for some $\mu_{1} \approx \mu, \mu_{1} \in \mathfrak{M}$ and $\mu_{1} \circ S \in \mathfrak{M}$ then $S=T^{i}$ for some $i$.

Proof. Let $\hat{\mu}$ be a joining supported on the graph of $S$, i.e.,

$$
\hat{\mu}(A \times B)=\mu_{1}(S(A) \cap B) .
$$


Thus $\hat{\mu}(\{(x, S(x)): x \in X\})=1$. This joining is rational since $\hat{\mu}_{x}$ is a point mass at $S(x)$ and $\hat{\mu}^{y}$ is a point mass at $S^{-1}(y)$. Clearly, the marginals on the first and second coordinates are $\mu_{1} \circ S$ and $\mu_{1}$, respectively. Therefore,

$$
\hat{\mu}=\alpha\left(\mu_{1} \circ S \times \mu_{1}\right)+\sum_{i=0}^{\infty} \alpha_{i} \delta_{i}
$$

where $\delta_{i}$ is a joining supported on $G_{i}$. Since $(X \times X, T \times T, \hat{\mu})$ is isomorphic to $(X, T, \mu)$, it is ergodic. Hence the ergodic decomposition above has only one term. This term must be one of the $\delta_{i}$ since $\hat{\mu}_{x}$ is a point mass. Thus $\hat{\mu}\left(\left\{\left(x, T^{i}(x)\right): x \in X\right\}\right)=$ 1 and so $S=T^{i}$.

Theorem 3.1.12. Suppose $(X, \mathscr{B}, \mu, T)$ is conservative totally ergodic and has minimal self-joinings over $\mathfrak{M}$. If $\mathfrak{M} \neq \varnothing$ then $T$ is prime.

Proof. Suppose $(X, \mu, T)$ has a nontrivial factor $\mathscr{D}$. Choose $A \in \mathscr{D}$ with $\mu(A) \neq 0,1$. Let $\hat{\mu}$ be the relatively independent joining of $(X, \mu)$ with itself over the factor algebra $\mathscr{D}$. Then

$$
\hat{\mu}=\alpha(\mu \times \mu)+\sum_{i=0}^{\infty} \alpha_{i} \delta_{i}
$$

where $\delta_{i}$ is the joining supported on $G_{i}$. Since $\hat{\mu}$ is a relatively independent joining, for $A \in \mathscr{D}, \hat{\mu}(A \times A)=\mu(A)$. So

$$
\hat{\mu}(A \times A)=\alpha \mu(A)^{2}+\sum_{i=0}^{\infty} \alpha_{i} \delta_{i}(A \times A) .
$$

By Lemma 3.1.7,

$$
\tilde{\mu}=\sum \frac{\alpha_{i}}{1-\alpha} \delta_{i}
$$

is a rational joining of $\mu$ with itself, in particular a joining. Thus $\tilde{\mu}(A \times A) \leq$ $\tilde{\mu}(A \times X)=\mu(A)$. We conclude

$$
\mu(A) \leq \alpha \mu(A)^{2}+(1-\alpha) \mu(A) .
$$

This implies $\alpha=0$. Define the projection $\pi_{1, i}:\left(x, T^{i}(x)\right) \rightarrow x$. Then

$$
\left.\sum \alpha_{i} \delta_{i} \circ \pi_{1, i}^{-1}\left(A \cap T^{-i}(A)\right)\right)=\sum \alpha_{i} \delta_{i} \circ \pi_{1, i}^{-1}(A) .
$$

Since each term on the left is $\leq$ each term on the right, if $\alpha_{i} \neq 0$ then $A=A \cap T^{-i}(A) \delta_{i} \circ \pi_{1, i}^{-1}$-a.e. Since all $\delta_{i} \circ \pi_{1, i}^{-1}$ are equivalent to $\mu$, if $\alpha_{i} \neq 0$ then $A=A \cap T^{-i}(A) \mu$-a.e. Since $A$ is nontrivial and $T$ totally ergodic, if $\alpha_{i} \neq 0$ then $i=0$. Thus $\hat{\mu}=\delta_{0}$ and for all sets $A \in \mathscr{B}, \hat{\mu}(A \times A)=\hat{\mu}(A \times X)=\mu(A)$. But it is well-known (cf. [R], [JR]) that this formula characterizes the factor algebra $\mathscr{D}$, and hence $\mathscr{D}=\mathscr{B}$.

We now show that the assumption of total ergodicity in Theorems 3.1.11 and 3.1.12 is simply that $X$ is nonatomic.

Proposition 3.1.13. Suppose $(X, \mathscr{B}, \mu, T)$ has minimal self-joinings over $\mathfrak{M}$. If $\mathfrak{M} \neq \varnothing$ and $T$ is not totally ergodic then $\mu$ is atomic. 
Proof. Minimal self-joinings implies $\mathrm{T}$ is ergodic. Since $\mathrm{T}$ is not totally ergodic there is an integer $n$ and a partition $\left\{A_{0}, A_{1}, \ldots, A_{n-1}\right\}$ of $X$ with $T\left(A_{i}\right)=A_{(i+1) \bmod n} \mu$-a.s. This partition forms an atomic $T$-invariant $\sigma$-algebra $\mathscr{D}$. Let $\hat{\mu}$ be the relatively independent joining of $\mu$ with itself over $\mathscr{D}$. The proof of Theorem 3.1.11 implies $\hat{\mu}=\sum a_{i} \delta_{i}$. Hence the fibre measures of $\hat{\mu}$ over $\mu$ are atomic. These though are the fibre measures of $\mu$ over $\mathscr{D}$. Hence $\mu$ restricted to $\mathscr{D}$ is atomic and relative to $\mathscr{D}$ is atomic. We conclude $\mu$ is atomic.

Question B. Using rational joinings one can define a notion of rational disjointness (cf. [F1]) for nonsingular transformations. Are weakly mixing or perhaps mildly mixing transformations rationally disjoint from nonsingular group rotations? This would answer a question of Aaronson's, who has asked the authors whether compact group rotations can have nontrivial weakly mixing factors (cf. [AN]).

\subsection{Graphic rational joinings}

We have seen that for any $\mu_{1}$ and $\mu_{2}$ product measure is a rational joining; also, for any $\mu_{1}$, if $\mu_{2}=\mu_{1} \circ T^{-j}$, then any average of product measure and $\mu_{1} \circ \pi_{1}$ is a rational joining of $\mu_{1}$ and $\mu_{2}$. We now want to argue that only under special circumstances can a graphic rational joining of $\mu_{1}$ and $\mu_{2}$ exist supported on more than one graph. We will first describe examples of how this might occur, and then we will see that these examples are the only ones.

First example. Suppose $\mu$ is an invariant probability measure for $T$. Let $\delta_{j}$ be the off-diagonal supported on $G_{j}=\left\{\left(x, T^{j} x\right)\right\}$. Any measure

$$
\hat{\mu}=\sum a_{j} \delta_{j}, \quad \text { where } a_{j}>0, \quad \sum a_{j}=1,
$$

is a graphic joining of $\mu$ with itself.

Under certain circumstances we can modify the marginals and joining above simultaneously to still be a rational joining. Here are those circumstances.

Suppose there exist $f, g \in L_{+}^{1}(\mu)$ and that they satisfy

$$
\sum_{i \in \mathbb{Z}} a_{i} f\left(T^{i}(x)\right)=1 \text { and } \sum_{i \in \mathbb{Z}} a_{i} g\left(T^{-i}(x)\right)=1 .
$$

This condition forces $\int f=\int g=1$, and $f, g \in L^{\infty}(\mu)$ since $f(x), g(x) \leq 1 /$ sup $a_{i}$.

Define a new measure $\tilde{\mu}$ supported on graphs $G_{j}$ by

$$
\frac{d \tilde{\mu}}{d \hat{\mu}}\left(x, T^{j}(x)\right)=g(x) f\left(T^{j}(x)\right) \in L^{\infty}(\hat{\mu}) .
$$

To see that $\tilde{\mu}$ is a probability measure we compute

$$
\begin{aligned}
\int_{X \times X} \frac{d \tilde{\mu}}{d \hat{\mu}} d \hat{\mu} & =\int_{X} \int_{\{x\} \times X} g(x) f\left(T^{j}(x)\right) d \hat{\mu}_{x} d \mu(x) \\
& =\int_{X}\left[\sum_{j \in \mathbb{Z}} a_{j} g(x) f\left(T^{j}(x)\right)\right] d \mu(x)=1 .
\end{aligned}
$$

Letting $\mu_{1}$ and $\mu_{2}$ be the marginals of $\tilde{\mu}$, from our last computation

$$
\frac{d \mu_{1}}{d \mu}(x)=g(x), \quad \frac{d \mu_{2}}{d \mu}(y)=f(y) .
$$


To see that $\tilde{\mu}$ is a rational joining of $\mu_{1}$ and $\mu_{2}$ note that

$$
\begin{aligned}
\frac{d \tilde{\mu} \circ(T \times T)}{d \tilde{\mu}}\left(x, T^{j}(x)\right) & =\frac{g(T x) f\left(T^{j+1}(x)\right)}{g(x) f\left(T^{j}(x)\right)} \\
& =\frac{d \mu_{1} \circ T}{d \mu_{1}}(x) \frac{d \mu_{2} \circ T}{d \mu_{2}}\left(T^{j}(x)\right) .
\end{aligned}
$$

It is natural to ask when the equations in condition (3.1) can have nonconstant solutions $f$ and g. Condition (3.1) is equivalent to asking for $f \in L^{\infty}(\mu)$ with 0 in the convex hull of the functions $f \circ T^{i}$ if we replace $f$ by $(f-\operatorname{ess} \inf f) /\left(\int f-\right.$ ess inf $f$ ). For $T$ an irrational rotation on the unit circle in $\mathbb{C}$, it is easy to see that $f(x)=z$ and hence $f(z)=\operatorname{re}(z)=\cos (\theta)$ satisfies an equation of the form (3.1). As $\cos (-\theta)=\cos (\theta)$, we can use $f=g$. Thus for any non-weakly mixing $T$, nontrivial versions of (3.1) hold. (We note that when $T$ is type III, (3.1) may also have a solution. In fact, in the case above of an irrational rotation, (3.1) holds everywhere, not just a.e., therefore also for a type III measure.)

In an earlier version of this paper we asked whether (3.1) holds for weakly mixing $T$. This question has been answered by J. F. Méla. We are indebted to Méla for the proof of the lemma below.

LeMma 3.2.1. (Méla.) Let $(X, \mu)$ be a probability space and $T$ an ergodic measure preserving transformation. Then there exist nonnegative constants $a_{j}, \sum a_{j}<\infty$, and a nonconstant $f \in L^{\infty}(\mu)$ with

$$
\sum_{i \in \mathbb{Z}} a_{i} f\left(T^{i}(x)\right)=0 \mu \text {-a.e. }
$$

Proof. Choose $f_{0} \in L^{\infty}(\mu)$ a nonconstant function. Hence $\sigma_{f_{0}}$, its spectral measure, has some support away from 0 . For $a>0$ let $h \in L^{2}\left(K, \sigma_{f_{0}}\right)$ be a 'tent' function with support on $(-a, a)$. Then $h(t)=\sum a_{j} \mathrm{e}^{2 \pi \mathrm{ij} t}$, where $a_{j}=a_{-j}$ as $h$ is real. A computation shows that $\sum\left|a_{j}\right|<\infty$. Choose $\alpha \in \operatorname{supp}\left(\sigma_{f_{0}}\right), \alpha \neq 0$, so that

$$
h_{\alpha}(t)=\sum a_{j} \mathrm{e}^{2 \pi \mathrm{ij} \alpha} \mathrm{e}^{2 \pi \mathrm{i} j t}=h(\alpha+t) \not \equiv 0 \sigma_{f_{0}} \text {-a.e. }
$$

Choose $a$ so that $h(t) h_{\alpha}(t)=0$ (i.e. make the two tents disjoint).

Let $Z\left(f_{0}\right)$ denote the cyclic subspace generated by $f_{0}$, and $W$ the isometry from $Z\left(f_{0}\right)$ onto $L^{2}\left(K, \sigma_{f_{0}}\right)$ taking $f_{0}$ to 1 and the action of $T$ to multiplication by $\mathrm{e}^{2 \pi \mathrm{i} j t}$. (cf. [P] for notation.) Let $f_{1}(x)=\sum a_{j} \mathrm{e}^{2 \pi \mathrm{i} j \alpha} f_{0}\left(T^{j} x\right)$. Then $f_{1} \in L^{\infty}(\mu)$ and $W\left(f_{1}\right)=h_{\alpha}$. Now

$$
\begin{aligned}
W\left(\sum a_{j} f_{1}\left(T^{j} x\right)\right) & =\sum a_{j} W\left(f_{1}\left(T^{j} x\right)\right)=\sum a_{j} \mathrm{e}^{2 \pi \mathrm{i} j t} W\left(f_{1}(x)\right) \\
& =\sum a_{j} \mathrm{e}^{2 \pi \mathrm{i} j t} h_{\alpha}(t)=h(t) h_{\alpha}(t)=0 \sigma_{f_{0}} \text {-a.e. }
\end{aligned}
$$

Therefore $\sum a_{j} f_{1}\left(T^{j} x\right)=0 \mu$-a.e. To make $f_{1}$ real just define

$$
f_{2}(x)=\sum a_{j} \mathrm{e}^{-2 \pi \mathrm{i} j \alpha} f_{0}\left(T^{j} x\right)
$$

supported on $(-\alpha-a,-\alpha+a)$. Then let $f=f_{1}+f_{2}$.

Second example. Suppose $\mu$ is an infinite, $\sigma$-finite invariant measure for $T$. We will see here also that non-trivial graphic rational joinings of measures equivalent to $\mu$ may exist. 
Suppose $\left\{a_{i}\right\}_{i \in \mathbb{Z}}$ is a sequence of nonnegative numbers. Again suppose $f, g \in L_{+}^{1}(\mu)$ satisfy

$$
\sum_{i \in \mathbb{Z}} a_{i} f\left(T^{i}(x)\right)=1 \text { and } \sum_{i \in \mathbb{Z}} a_{i} g\left(T^{-i}(x)\right)=1 .
$$

Since $\mu$ is infinite, these imply $\sum a_{i}=\infty$.

Condition (3.2) implies $f, g \in L^{\infty}(\mu)$, and we normalize them so that $\int f=\int g=1$, and then normalize the $\left\{a_{i}\right\}$ to maintain (3.2).

If we define a joining $\hat{\mu}=\sum a_{j} \delta_{j}$, where $\delta_{j}$ is the off-diagonal on $G_{j}$, we get a $\sigma$-finite $T \times T$-invariant measure. It is not a joining of $\mu$ with itself as both marginals give infinite measure to all sets of $\mu$-positive measure. We can modify $\hat{\mu}$ to $\tilde{\mu}$ just as in example 1 to give a rational joining. In fact, define $\tilde{\mu}$ by

$$
\frac{d \tilde{\mu}}{d \hat{\mu}}\left(x, T^{j}(x)\right)=g(x) f\left(T^{j}(x)\right) .
$$

We compute

$$
\begin{aligned}
\int_{X \times X} g(x) f(y) d \hat{\mu} & =\sum_{j} \int_{G_{j}} g(x) f\left(T^{j}(x)\right) d \hat{\mu} \\
& =\sum_{j} a_{i} \int_{X} g(x) f\left(T^{j}(x)\right) d \mu .
\end{aligned}
$$

Now $g \in L_{+}^{1}(\mu)$ and $f \in L^{\infty}(\mu)$ so $g f \circ T^{j} \in L_{+}^{1}(\mu)$. By monotone convergence,

$$
=\int_{X} \sum_{j} a_{j} g(x) f\left(T^{j}(x)\right) d \mu=1 .
$$

Thus $\tilde{\mu}$ is a probability measure equivalent to $\hat{\mu}$. To compute its marginals $\mu_{1}$ and $\mu_{2}$,

$$
\begin{aligned}
\mu_{1}(A) & =\tilde{\mu}(A \times X)=\int_{A \times X} g(x) f(y) d \hat{\mu} \\
& =\sum_{j} \int_{A} g(x) f\left(T^{j}(x)\right) d \hat{\mu} \\
& =\sum_{j} a_{j} \int_{A} g(x) f\left(T^{j}(x)\right) d \mu(x) \\
& =\int_{A} g(x) d \mu(x), \quad \text { and } \\
\mu_{2}(A) & =\tilde{\mu}(X \times A)=\int_{X \times A} g(x) f(y) d \hat{\mu} \\
& =\sum_{j} \int_{A} g\left(T^{-j}(y)\right) f(y) d \hat{\mu} \\
& =\sum_{j} a_{j} \int_{A} g\left(T^{-j}(y)\right) f(y) d \mu(y) \\
& =\int_{A} f(y) d \mu(y) .
\end{aligned}
$$


Thus

$$
\frac{d \mu_{1}}{d \mu}(x)=g(x) \text { and } \frac{d \mu_{2}}{d \mu}(y)=f(y) .
$$

Just as in Example 1,

$$
\begin{aligned}
\frac{d \tilde{\mu} \circ(T \times T)}{d \tilde{\mu}}\left(x, T^{j}(x)\right) & =\frac{g(T x) f\left(T^{j+1}(x)\right)}{g(x) f\left(T^{j}(x)\right)} \\
& =\frac{d \mu_{1} \circ T}{d \mu_{1}}(x) \frac{d \mu_{2} \circ T}{d \mu_{2}}\left(T^{j}(x)\right),
\end{aligned}
$$

and $\tilde{\mu}$ is a rational joining of $\mu_{1}$ and $\mu_{2}$.

In the infinite measure preserving case, the possible existence of nontrivial $\left\{a_{i}\right\}_{i \in \mathbb{Z}}, f$ and $g$ is easy to see.

Let $\boldsymbol{A}$ be an exhaustive weakly wandering set of finite measure [JK], i.e. $\mu(A)<\infty$, and there exist $j(i)$ with $T^{j(i)}(A)$ disjoint and covering $X$. Let $f_{0}=\chi_{A}$ and

$$
a_{j}=1 \text {, if } j=-j(i) \text {, for some } i, \quad \text { and } a_{j}=0 \text { otherwise. }
$$

For a.e. $x \in X$ there is a unique $i(x)$ with $x \in T^{j(i(x))}(A)$. Thus

$$
f_{0}\left(T^{-j(i(x))} x\right)=1 \text {. }
$$

Thus

$$
\sum_{i \in \mathbb{Z}} a_{i} f_{0}\left(T^{i}(x)\right)=1 \text { a.e. }
$$

To get $f \in L_{+}^{1}(\mu)$ let

$$
f(x)=\frac{1}{3} \sum_{j=-\infty}^{\infty} \frac{1}{2^{|j|}} f_{0}\left(T^{j}(x)\right) .
$$

Now $f>0$ a.e. as $T$ is ergodic. We still have $\int f(x) d \mu=1$ and

$$
\sum_{i \in \mathbb{Z}} a_{i} f\left(T^{i}(x)\right)=1 \text { a.e. }
$$

Since $A$ has finite measure, the sets $T^{-j(i)}(A)$ are also disjoint and cover $X$ [EIH], hence $f=g$ solves $\sum a_{i} g\left(T^{i}(x)\right)=1$.

Question $C$. There are infinite measure preserving systems whose weakly wandering exhaustive sets must have infinite measure [HK], [HIK]. Can solutions still exist in this case?

Proposition 3.2.2. Suppose $T$ is conservative and totally ergodic and $\tilde{\mu}$ is a graphic rational joining of $\mu_{1}$ and $\mu_{2}$. If $\tilde{\mu}$ is supported on more than one graph, then $\tilde{\mu}$ is of the form of either Example 1 or Example 2 above.

The proof proceeds in a series of lemmas.

LEMMA 3.2.3. Suppose $T$ is conservative and totally ergodic and $\tilde{\mu}$ is a graphic rational joining of $\mu_{1}$ and $\mu_{2}$. If $\tilde{\mu}$ is supported on more than one graph, then there exist $\sigma$-finite invariant measures $\mu_{i}^{0}$ equivalent to $\mu_{1}$. Furthermore, they are finite or infinite for all $i$, where $i$ varies over the graphs. 
Proof. Since $T$ is totally ergodic, the $G_{j}=\left\{\left(x, T^{j}(x)\right): x \in X\right\}$ are $\tilde{\mu}$-a.s. disjoint, and $T \times T$-invariant. Let $G_{j(1)}, G_{j(2)}, \ldots$, be the graphs of nonzero $\tilde{\mu}$-measure. Define

$$
\tilde{\mu}_{i}(A)=\frac{\tilde{\mu}\left(G_{j(i)} \cap A\right)}{\tilde{\mu}\left(G_{j(i)}\right)} .
$$

Since $\tilde{\mu}=\sum \tilde{\mu}\left(G_{j(i)}\right) \tilde{\mu}_{i}$, by Lemma 3.1.4, all the marginals $\tilde{\mu}_{i}^{1}$ and $\tilde{\mu}_{i}^{2}$ are absolutely continuous with respect to $\mu_{1}$ and $\mu_{2}$ respectively.

The projections of $\tilde{\mu}_{i}$ to the first and second coordinates are measure preserving to $\tilde{\mu}_{i}^{1}$ and $\tilde{\mu}_{i}^{2}$ and $1-1$.

Hence the inverse maps

$$
\pi_{1, i}^{-1}: x \rightarrow\left(x, T^{j(i)}(x)\right), \quad \pi_{2, i}^{-1}: x \rightarrow\left(T^{-j(i)}(x), x\right)
$$

are measure preserving $\tilde{\mu}_{i}^{j} \rightarrow \tilde{\mu}_{i}$, and so nonsingular from

$$
\left(X, \mu_{1}\right) \rightarrow\left(\tilde{\mu}_{i}, G_{j(i)}\right) \text { and }\left(X, \mu_{2}\right) \rightarrow\left(\tilde{\mu}_{i}, G_{j(i)}\right) \text {. }
$$

We are assuming $\tilde{\mu}$ is graphic and that at least two $G_{j(i)}$ exist. The fibre measure $\tilde{\mu}_{x}=\mathbb{E}_{\hat{\mu}}\left[\mathscr{B}_{2} \mid \mathscr{B}_{1}\right](x)$ is supported on the at most countable set $T^{j(1)}(x), T^{j(2)}(x), \ldots$, hence is atomic. The mass

$$
\tilde{\mu}_{x}\left(T^{j(i)}(x)\right)=\mathbb{E}_{\hat{\mu}}\left[\mathscr{B}_{2} \mid \mathscr{B}_{1}\right](x)=\frac{d \tilde{\mu}_{i}^{1}}{d \mu_{1}}(x) \tilde{\mu}\left(G_{j(i)}\right)>0 \text { for } \mu_{1} \text {-a.e. } x \text {. }
$$

Also, since the $G_{j(i)}$ are disjoint and $T \times T$-invariant, the $\tilde{\mu}_{i}$ are mutually singular and so

$$
\frac{d \tilde{\mu}_{i} \circ(T \times T)}{d \tilde{\mu}_{i}}\left(x, T^{j(i)}(x)\right)=\frac{d \tilde{\mu} \circ(T \times T)}{d \tilde{\mu}}\left(x, T^{j(i)}(x)\right) .
$$

Since $\tilde{\mu}$ is rational we know that $\tilde{\mu}$-a.e.,

$$
\frac{d \tilde{\mu} \circ(T \times T)}{d \tilde{\mu}}(x, y)=\frac{d \mu_{1} \circ T}{d \mu_{1}}(x) \frac{d \mu_{2} \circ T}{d \mu_{2}}(y) c(x, y),
$$

where $c$ is a.s. a constant on vertical and horizontal fibres of $\tilde{\mu}$. Thus $c\left(x, T^{j(i)}(x)\right)$ is defined for $\tilde{\mu}_{i}$-a.e. $x$ and its value is independent of $i$. Similarly $c\left(T^{-j(i)}(x), x\right)$ is defined for $\mu_{2}$-a.e. $x$ and its value is independent of $i$. Thus for $\mu_{1}$-a.e. $x$, if we let $c_{s}(x)=c\left(x, T^{j(s)}(x)\right)$, then

$$
\begin{aligned}
c_{s}(x) & =c\left(x, T^{j(t)}(x)\right) \\
& =c\left(T^{j(t)-j(s)}(x), T^{j(t)-j(s)}\left(T^{j(s)}(x)\right)\right. \\
& =c_{s}\left(T^{j(t)-j(s)}(x)\right) .
\end{aligned}
$$

As we can select $j(t)-j(s) \neq 0$ and since $T$ is totally ergodic, $c_{s}(x)$ is a constant $\mu_{1}$-a.e. We already saw $c_{s}(x)=c_{t}(x)$ so $c(x, y)$ is a constant $c, \tilde{\mu}$-a.e. Thus for all $j(i)$ and $\mu_{1}$-a.e. $x$

$$
\frac{d \tilde{\mu}_{i} \circ(T \times T)}{d \tilde{\mu}_{i}}\left(x, T^{j(i)}(x)\right)=\frac{d \mu_{1} \circ T}{d \mu_{1}}(x) \frac{d \mu_{2} \circ T}{d \mu_{2}}\left(T^{j(i)}(x)\right) c .
$$

Remember $\tilde{\mu}_{i} \circ \pi_{1 i}^{-1}$ is absolutely continuous with respect to $\mu_{2}$. Let

$$
f_{i}(y)=\frac{d \tilde{\mu}_{i} \circ \pi_{2 i}^{-1}}{d \mu_{2}}(y)=\frac{\mathbb{E}_{\hat{\mu}}\left[G_{j(i)} \mid \mathscr{B}_{2}\right]}{\mu\left(G_{j(i)}\right)}\left(T^{-j(i)}(y), y\right)
$$


and similarly

$$
g_{i}(x)=\frac{d \tilde{\mu}_{i} \circ \pi_{1 i}^{-1}}{d \mu_{1}}(y)=\frac{\mathbb{E}_{\hat{\mu}}\left[G_{j(i)} \mid \mathscr{B}_{1}\right]}{\mu\left(G_{j(i)}\right)}\left(T^{-j(i)}(x), x\right)
$$

Computing

$$
\begin{aligned}
\frac{d \tilde{\mu}_{i} \circ(T \times T)}{d \tilde{\mu}_{i}}\left(x, T^{j(i)}(x)\right) & =\frac{d \tilde{\mu}_{i} \circ \pi_{2 i}^{-1} \circ T}{d \tilde{\mu}_{i} \circ \pi_{1 i}^{-1}}\left(T^{j(i)}(x)\right) \\
& =\frac{f_{i}\left(T^{j(i)+1}(x)\right)}{f_{i}\left(T^{j(i)}(x)\right)} \frac{d \mu_{2} \circ T}{d \mu_{2}}\left(T^{j(i)}(x)\right) c .
\end{aligned}
$$

Thus

$$
\frac{d \mu_{1} \circ T}{d \mu_{1}}(x)=\frac{f_{i}\left(T^{j(i)}(T x)\right)}{c f_{i}\left(T^{j(i)}(x)\right)}
$$

For all $k$ we conclude

$$
\omega_{k}^{1}(x)=\frac{d \mu_{1} \circ T^{k}}{d \mu_{1}}(x)=\frac{f_{i}\left(T^{j(i)}\left(T^{k} x\right)\right)}{c^{k} f_{i}\left(T^{j(i)}(x)\right)} .
$$

Since $T$ is conservative and $f_{i} \in L^{\infty}(\mu)$, we know that there are arbitrarily large values of $k$ with

$$
\begin{aligned}
& \frac{1}{2} \leq \omega_{k}^{1}(x) \leq 2 \text { and } \\
& \frac{1}{2} \leq \frac{f_{i}\left(T^{j(i)}\left(T^{k} x\right)\right)}{f_{i}\left(T^{j(i)}(x)\right)} \leq 2 .
\end{aligned}
$$

But then $\frac{1}{4} \leq c^{k} \leq 4$ and hence $c=1$. For all $i$ then

$$
\frac{d \mu_{1} \circ T}{d \mu_{1}}(x)=\frac{f_{i}\left(T^{j(i)}(T x)\right)}{f_{i}\left(T^{j(i)}(x)\right)} \text {. }
$$

Symmetrically

$$
\frac{d \mu_{2} \circ T}{d \mu_{2}}(y)=\frac{g_{i}\left(T^{-j(i)}(T y)\right)}{g_{i}\left(T^{-j(i)}(y)\right)} .
$$

Defining

$$
\mu_{0}^{i}=\int \frac{1}{f_{i}\left(T^{j(i)}(x)\right)} d \mu_{1}
$$

$\mu_{0}^{i}$ is an invariant measure equivalent to $\mu_{1}$. It must be either finite or $\sigma$-finite for all $i$.

LEMMA 3.2.4. Under the assumptions of Lemma 3.2.3, suppose the measures $\mu_{i}^{0}$ are finite for all $i$. Then $\tilde{\mu}$ is of the form of Example 1.

Proof. Since the $\mu_{i}^{0}$ are finite, $\left(f_{i}\left(T^{j(i)}(x)\right)^{-1} \in L^{1}\left(\mu_{1}\right)\right.$. Let $c_{i}=\int\left(f_{i}\left(T^{j(i)}(x)\right)^{-1} d_{\mu_{1}}\right.$, and define

$$
\mu_{0}=\int \frac{1}{c_{i} f_{i}\left(T^{j(i)}(x)\right)} d \mu_{1}
$$


This is a $T$-invariant probability measure, hence is unique. We conclude $c_{i} f_{i}\left(T^{j(i)}(x)\right)$ is independent of $i$. Computing

$$
\begin{aligned}
\frac{d \mu_{1}}{d \mu_{2}}(x) & =\frac{d \mu_{1}}{d \tilde{\mu}_{k} \circ \pi_{1 i}^{-1}}(x) \frac{d \tilde{\mu}_{k} \circ \pi_{2 k}^{-1}}{d \mu_{2}}\left(T^{j(i)}(x)\right) \frac{d \mu_{2} \circ T^{j(k)}}{d \mu_{2}}(x) \\
& =\frac{f_{k}\left(T^{j(k)}(x)\right)}{g_{k}(x)} \frac{g_{k}(x)}{g_{k}\left(T^{-j(k)}(x)\right)}=\frac{f_{k}\left(T^{j(k)}(x)\right)}{g_{k}\left(T^{-j(k)}(x)\right)}
\end{aligned}
$$

we obtain

$$
c_{k}=\int \frac{1}{f_{k}\left(T^{j(k)}(x)\right)} d \mu_{1}(x)=\int \frac{1}{g_{k}\left(T^{-j(k)}(x)\right)} d \mu_{2}(x) .
$$

Thus

$$
\mu_{0}=\int \frac{1}{c_{k} g_{k}\left(T^{-j(k)}(x)\right)} d \mu_{2} .
$$

Since $\left.\left.\tilde{\mu}=\sum_{i} \tilde{\mu}\left(G_{j(i)}\right)\right) \tilde{\mu}_{i}, \mu_{2}=\sum_{i} \tilde{\mu}\left(G_{j(i)}\right)\right) \tilde{\mu}_{i} \circ \pi_{2 k}^{-1}$ and so

$$
\left.1=\sum_{i} \tilde{\mu}\left(G_{j(i)}\right)\right) f_{i}(x) \text {. }
$$

But we know, for all $i$,

$$
c_{i} f_{i}\left(T^{j(i)}(x)\right)=c_{1} f_{1}\left(T^{j(1)}(x)\right)
$$

Thus

$$
\left.1=\sum_{i} \tilde{\mu}\left(G_{j(i)}\right)\right)\left(\frac{c_{1}}{c_{i}}\right) f_{1}\left(T^{-j(i)}(x)\right)
$$

Symmetrically

$$
\left.1=\sum_{i} \tilde{\mu}\left(G_{j(i)}\right)\right)\left(\frac{c_{1}}{c_{i}}\right) g_{1}\left(T^{-j(i)}(x)\right)
$$

Setting

we obtain

$$
a_{j}= \begin{cases}\frac{\tilde{\mu}\left(G_{j(i)}\right)}{c_{i}} & \text { if } j=j(i) \\ 0 & \text { otherwise }\end{cases}
$$

$$
\begin{gathered}
\sum_{j} a_{j} c_{1} f_{1}\left(T^{-j+j(1)}(x)\right)=1 \quad \text { and } \\
\sum_{j} a_{j} c_{1} g_{1}\left(T^{j-j(1)}(x)\right)=1 .
\end{gathered}
$$

Since

$$
1=\int \frac{f_{k}\left(T^{j(k)}(x)\right)}{g_{k}\left(T^{-j(k)}(x)\right)} d \mu_{2}=\int c_{k} f_{k}\left(T^{j(k)}(x)\right) d \mu_{0}
$$

we conclude $f(x)=c_{1} g_{1}\left(T^{-j(1)}(x)\right)$ and $g(x)=c_{1} f_{1}\left(T^{-j(1)}(x)\right)$ satisfy (3.2).

Define $\hat{\mu}$ on $G_{j(i)}$ by

$$
\frac{d \hat{\mu}}{d \hat{\mu}}\left(x, T^{j(i)}(x)\right)=\frac{1}{g(x) f\left(T^{j(i)}(x)\right)}=\frac{1}{c_{i}^{2} f_{i}\left(T^{j(i)}(x)\right) g_{i}(x)} .
$$

We compute that $\hat{\mu}$ is a probability measure. 


$$
\begin{aligned}
\int_{X \times X} d \hat{\mu} & =\sum_{i} \tilde{\mu}\left(G_{j(i)}\right) \int \frac{\tilde{\mu}_{i}\left(x, T^{j(i)}(x)\right)}{c_{i}^{2} f_{i}\left(T^{j(i)}(x)\right) g_{i}(x)} d \mu_{2} \\
& =\sum_{i} \frac{\tilde{\mu}\left(G_{j(i)}\right)}{c_{i}} \int \frac{d \mu_{1}(x)}{c_{i} f_{i}\left(T^{j(i)}(x)\right)} \\
& =\sum_{i} \frac{\tilde{\mu}\left(G_{j(i)}\right)}{c_{i}} \int d \mu_{0}(x)=1 .
\end{aligned}
$$

It is $T \times T$ invariant since

$$
\frac{d \hat{\mu} \circ(T \times T)}{d \hat{\mu}}\left(x, T^{j}(x)\right)=\frac{d \tilde{\mu} \circ(T \times T)}{d \tilde{\mu}} \frac{g_{i}(x) f_{i}\left(T^{j(i)}(x)\right)}{g_{i}(T(x)) f_{i}\left(T^{j(i)+1}(x)\right)}=1 .
$$

Thus its marginals must both be $\mu_{0}$.

Arguing backwards,

$$
\frac{d \tilde{\mu}}{d \hat{\mu}}\left(x, T^{j}(x)\right)=g(x) f\left(T^{j(i)}(x)\right)
$$

is of the form of example 1 .

LEMMA 3.2.5. Under the assumptions of Lemma 3.2.3, suppose the measures $\mu_{i}^{0}$ are infinite for all $i$. Then $\tilde{\mu}$ is of the form of Example 2.

Proof. As in Lemma 3.2.4, since $\mu_{0}$ is essentially unique,

$$
f_{i}\left(T^{j(i)}(x)\right)=c_{i} f_{1}\left(T^{j(1)}(x)\right)
$$

and since

$$
\frac{d \mu_{1}}{d \mu_{2}}(x)=\frac{f_{k}\left(T^{j(k)}(x)\right)}{g_{k}\left(T^{-j(k)}(x)\right)}, \quad g_{i}\left(T^{-j(i)}(x)\right)=c_{i} g_{1}\left(T^{-j(1)}(x)\right)
$$

Let

$$
\mu_{0}=\mu_{0}^{1}=\int \frac{1}{f_{1}\left(T^{j(1)}(x)\right)} d \mu_{1},
$$

just to normalize the selection. Just as in the previous case,

$$
\begin{aligned}
& 1=\sum_{i} \frac{\tilde{\mu}\left(G_{j(i)}\right)}{c_{i}}\left(c_{i} f_{i}(x)\right) \quad \text { and } \\
& 1=\sum_{i} \frac{\tilde{\mu}\left(G_{j(i)}\right)}{c_{i}}\left(c_{i} g_{i}(x)\right) .
\end{aligned}
$$

Set

$$
a_{j}= \begin{cases}\frac{\tilde{\mu}\left(G_{j(i)}\right)}{c_{i}} & \text { if } j=j(i), \\ 0 & \text { otherwise }\end{cases}
$$

and $f(x)=g_{1}\left(T^{-j(i)}(x)\right), g(x)=f_{1}\left(T^{j(i)}(x)\right)$, and we conclude. 
Now

$$
\frac{f(x)}{g(x)}=\frac{g_{1}\left(T^{-j(1)}(x)\right)}{f_{1}\left(T^{j(1)}(x)\right)}(x)=\frac{d \mu_{1}}{d \mu_{2}}(x),
$$

so

$$
\int \frac{f(x)}{g(x)} d \mu_{1}(x)=1 .
$$

But it also is $\int f(x) d \mu_{0}$. Hence $f$, and symmetrically $g$, belong to $L_{+}^{1}\left(\mu_{0}\right)$, with normed integral. Defining $\hat{\mu}_{i}$ on $G_{j(i)}$ by

$$
\frac{d \hat{\mu}_{i}}{d \tilde{\mu}}\left(x, T^{j(i)}(x)\right)=\frac{1}{c_{i} g(x) f\left(T^{j(i)}(x)\right)}=\frac{1}{c_{i} f_{i}\left(T^{j(i)}(x)\right) g_{i}(x)} .
$$

We compute $\hat{\mu}_{i}$ is $T \times T$ invariant, just as for the previous example. Also,

$$
\begin{aligned}
\frac{d \hat{\mu}_{i} \circ \pi_{1 i}^{-1}}{d \mu_{0}}(x) & =\frac{d \hat{\mu}_{i}}{d \tilde{\mu}}\left(x, T^{j(i)}(x)\right) \frac{d \tilde{\mu} \circ \pi_{1 i}^{-1}}{d \mu_{1}}(x) \frac{d \mu_{1}}{d \mu_{0}}(x) \\
& =\frac{1}{c_{i} f_{i}\left(T^{j(i)}(x)\right) g_{i}(x)}\left(g_{i}(x)\right)\left(c_{i} f_{i}\left(T_{j(i)}(x)\right)\right) \\
& =1 .
\end{aligned}
$$

Thus $\hat{\mu}_{i}=\mu_{0} \circ \pi_{1 i}^{-1}$. Setting

$$
\hat{\mu}=\sum a_{j(i)} \hat{\mu}_{i}=\sum_{i} \frac{\hat{\mu}\left(G_{j(i)}\right)}{c_{i}} \hat{\mu}_{i}
$$

we conclude that on $G_{j(i)}$,

$$
\frac{d \tilde{\mu}}{d \hat{\mu}}\left(x, T^{j}(x)\right)=\frac{1}{f\left(T^{j(i)}(x)\right) g(x)}
$$

and so is of the form of Example 2.

Thus whenever a graphic rational joining is supported on at least two graphs, the joining is equivalent to a weighted average of off-diagonal measures for some finite or infinite invariant measure, completing the proof of Proposition 3.2.2.

\section{Examples}

Our next task is to construct examples of systems of various Krieger types with minimal self-joinings. These arguments owe much to [JRS] who show Chacón's map has minimal self-joinings.

4.1. $\alpha$-matching structures

Let $\left(X_{1}, \mathscr{B}_{1}, \mu_{1}, T_{1}\right)$ and $\left(X_{2}, \mathscr{B}_{2}, \mu_{2}, T_{2}\right)$ be two ergodic dynamical systems and $\hat{\mu}$ a conservative nonsingular joining of $\mu_{1}$ and $\mu_{2}$. Furthermore, suppose $\left\{P_{i}\right\}$ and $\left\{Q_{i}\right\}$ are refining and generating sequences of partitions on $X_{1}$ and $X_{2}$. Let $A \subset X_{1} \times X_{2}$, with $\hat{\mu}(A)>0$, and $0<\alpha<1$. An $\alpha$-matching structure on $A$ (for $\left.\left\{\left(X_{1}, \mathscr{B}_{1}, \mu_{1}, T_{1}\right),\left(X_{2}, \mathscr{B}_{2}, \mu_{2}, T_{2}\right),\left\{P_{i}\right\},\left\{Q_{i}\right\}, \hat{\mu}\right\}\right)$ is the following. For any $(x, y) \in A$ there exist $i_{n}=i_{n}(x, y), j_{n}=j_{n}(x, y) \in \mathbb{Z}$, with

$$
i_{n} \leq 0 \leq j_{n}, j_{n}-i_{n} \gg \infty \text { in } n,
$$

and subsets $B_{n}, B_{n}^{\prime} \subset\left[i_{n}, j_{n}\right]$, and a bijection $\pi_{n}: B_{n} \rightarrow B_{n}^{\prime}$ so that: 
(i) both $B_{n}$ and $B_{n}^{\prime}$ are disjoint unions of intervals each of length $\geq n$,

$$
\sum_{i \in B_{n}} \hat{\omega}_{i}(x, y) \geq \alpha \sum_{i=i_{n}}^{j_{n}} \hat{\omega}_{i}(x, y)
$$

and

$$
\sum_{i \in B_{n}^{\prime}} \hat{\omega}_{i}(x, y) \geq \alpha \sum_{i=i_{n}}^{j_{n}} \hat{\omega}_{i}(x, y)
$$

(iii) for $i \in B_{n}$,

$$
\frac{1}{\alpha} \geq \frac{\hat{\omega}_{i}(x, y)}{\hat{\omega}_{\pi(i)}(x, y)} \geq \alpha
$$

(iv) for $j \in B_{n}, T_{1}^{j}(x) \in P_{n, l}$ iff $T_{1}^{\pi_{n}(j)}(x) \in P_{n, l}$, and

$$
T_{2}^{j}(x) \in Q_{n, l} \text { iff } T_{2}^{\pi_{n}(j)-1}(x) \in Q_{n, l} .
$$

(Here $P_{n, l}$ denotes the $l$ th element of the partition $P_{n}$.)

Notice that the set $A$ of the $\alpha$-matching structure can be assumed $T_{1} \times T_{2}$ invariant, hence is a union of ergodic components of $\hat{\mu}$. (If $i_{n}(x, y)$ and $j_{n}(x, y)$ exist then these same translated by 1 will also work for $\left(T_{1}(x), T_{2}(x)\right)$, perhaps adding one further index if $j_{n}(x)$ is 0 .)

Our next proposition concerns these ergodic components. (Notice that rationality is not needed.)

Proposition 4.1.1. Let $\left\{\left(X_{1}, \mathscr{B}_{1}, \mu_{1}, T_{1}\right),\left(X_{2}, \mathscr{B}_{2}, \mu_{2}, T_{2}\right),\left\{P_{i}\right\},\left\{Q_{i}\right\}, \hat{\mu}\right\}$ be as above. If for some $\alpha>0$ there is $a T_{1} \times T_{2}$ invariant set $A$ and an $\alpha$-matching structure on $A$, then for a.e. ergodic component $\nu_{z}$ of $\hat{\mu}$ contained in $A, \nu_{z}$ is $I \times T_{2}$ nonsingular.

We need a preliminary lemma. From now on we assume a fixed $\alpha$-matching structure exists on a set $A$.

LEMMA 4.1.2. For any $f \in L_{+}^{\infty}(\hat{\mu})$, for $\hat{\mu}$-a.e. $(x, y) \in A$,

$$
\lim _{n \rightarrow \infty} \frac{\sum_{i \in B_{u}} f\left(\left(T_{1} \times T_{2}\right)^{i}(x, y)\right) \hat{\omega}_{i}(x, y)}{\sum_{i \in B_{n}} \hat{\omega}_{i}(x, y)}=\mathbb{E}_{\hat{\mu}}[f \mid \mathscr{I}](x, y)
$$

and

$$
\lim _{n \rightarrow \infty} \frac{\sum_{i \in B_{i}} f\left(\left(T_{1} \times T_{2}\right)^{i}(x, y)\right) \hat{\omega}_{i}(x, y)}{\sum_{i \in B_{i}^{\prime}} \hat{\omega}_{i}(x, y)}=\mathbb{E}_{\hat{\mu}}[f \mid \mathscr{I}](x, y) .
$$

Proof. We argue the first limit. Fix $\varepsilon>0$ and select $N_{0}$ so that for $n>N_{0}$, and a set $G, \hat{\mu}(G)>1-\varepsilon^{2}$, if $(x, y) \in G$ and $i \leq 0 \leq j, j-i>n$,

$$
\frac{\sum_{k=i}^{j} f\left(\left(T_{1} \times T_{2}\right)^{k}(x, y)\right) \hat{\omega}_{k}(x, y)}{\sum_{k=i}^{j} \hat{\omega}_{k}(x, y)}=\mathbb{E}_{\hat{\mu}}[f \mid \mathscr{I}](x, y) \pm \varepsilon .
$$

For all but a set of ergodic components $\nu_{z}$ of $\hat{\mu}$ of $\hat{\mu}$-measure $\leq \varepsilon, \nu_{z}(G)>1-\varepsilon$, since $\int \nu_{z}(G) d \hat{\mu}(z)=\hat{\mu}(G)$.

Thus for $(x, y)$ in a set $A(\varepsilon) \subset A, \hat{\mu}(A(\varepsilon))>\hat{\mu}(A)-\varepsilon$,

$$
\lim _{n \rightarrow \infty} \frac{\sum_{i=i_{u}(x, y)}^{j_{j}(x, y)} \chi_{G}\left(\left(T_{1} \times T_{2}\right)^{i}(x, y)\right) \hat{\omega}_{i}(x, y)}{\sum_{i=i_{n}(x, y)}^{j_{n}(x, y)} \hat{\omega}_{i}(x, y)}>1-\varepsilon .
$$


Hence by (ii) of $\alpha$-matching,



We assume $n>N_{0}$ so that $B_{n}$ consists of intervals of length $\geq n$. Let $B_{n}(\varepsilon) \subset B_{n}$ consist of those indices in an interval of length $\geq n$ which also contain a point of $G$. Now

$$
\frac{\sum_{i \in B_{n}(\varepsilon)} f\left(\left(T_{1} \times T_{2}\right)^{i}(x, y)\right) \hat{\omega}_{i}(x, y)}{\sum_{i \in B_{n}(\varepsilon)} \hat{\omega}_{i}(x, y)}=\mathbb{E}_{\hat{\mu}}[f \mid \mathscr{I}](x, y) \pm \varepsilon,
$$

(just average over the intervals).

Now for $(x, y) \in A(\varepsilon)$,

$$
\lim _{n \rightarrow \infty} \sup _{\inf } \frac{\sum_{i \in B_{n}} f\left(\left(T_{1} \times T_{2}\right)^{i}(x, y)\right) \hat{\omega}_{i}(x, y)}{\sum_{i \in B_{n}} \hat{\omega}_{i}(x, y)}
$$

is between

$$
\left(\mathbb{E}_{\hat{\mu}}[f \mid \mathscr{I}](x, y)-\varepsilon\right)\left(\frac{\sum_{i \in B_{n}(\varepsilon)} \hat{\omega}_{i}(x, y)}{\sum_{i \in B_{n}} \hat{\omega}_{i}(x, y)}\right)
$$

and

$$
\left(\mathbb{E}_{\hat{\mu}}[f \mid \mathcal{I}](x, y)+\varepsilon\right)+\left(\frac{\|f\|_{\propto}\left(\sum_{i \in B_{n} \backslash B_{u}(\varepsilon)} \hat{\omega}_{i}(x, y)\right)}{\sum_{i \in B_{n}} \hat{\omega}_{i}(x, y)}\right)
$$

hence between

$$
\left(\mathbb{E}_{\hat{\mu}}[f \mid \mathscr{I}](x, y)-\varepsilon\right)\left(1-\frac{\varepsilon}{\alpha}\right) \text { and } \mathbb{E}_{\hat{\mu}}[f \mid \mathscr{I}](x, y)+\varepsilon+\|f\|_{\infty} \frac{\varepsilon}{\alpha} .
$$

This completes the proof.

Corollary 4.1.3. For any set $C \in P_{k} \times Q_{k},\left(I \times T_{2}(C)=C^{\prime} \in P_{k} \times T_{2}\left(Q_{k}\right)\right.$, and for a.e. ergodic component $\nu_{z}, z \subset A$,

$$
\alpha^{2} \nu_{z}(C) \leq \nu_{z}\left(C^{\prime}\right)<\frac{\nu_{z}(C)}{\alpha^{2}} .
$$

Proof. Let $f_{1}(x, y)=\chi_{C}(x, y)$ and $f_{2}(x, y)=\chi_{C^{\prime}}(x, y)$. Notice that if $n>k$ and $(x, y) \in A, j \in B_{n}$ then

$$
f_{1}\left(\left(T_{1} \times T_{2}\right)^{j}(x, y)\right)=f_{2}\left(\left(T_{1} \times T_{2}\right)^{\pi(j)}(x, y)\right) .
$$

For $\hat{\mu}$-a.e. $\nu_{z}, z \subset A$, and $(x, y) \in z$,

$$
\frac{\nu_{z}(C)}{\nu_{z}\left(C^{\prime}\right)}=\lim _{n \rightarrow \infty} \frac{\sum_{j \in B_{n}} f_{1}\left(\left(T_{1} \times T_{2}\right)^{j}(x, y)\right) \hat{\omega}_{j}(x, y) \sum_{j \in B_{n}^{\prime}} \hat{\omega}_{j}(x, y)}{\sum_{j \in B_{n}^{\prime}} f_{2}\left(\left(T_{1} \times T_{2}\right)^{j}(x, y)\right) \hat{\omega}_{j}(x, y) \sum_{j \in B_{n}} \hat{\omega}_{j}(x, y)},
$$

by Lemma 4.1.2. But this equals,

$$
\lim _{n \rightarrow \infty}\left(\frac{\sum_{j \in B_{n}} f_{1}\left(\left(T_{1} \times T_{2}\right)^{j}(x, y)\right) \hat{\omega}_{j}(x, y)}{\sum_{j \in B_{n}} f_{1}\left(\left(T_{1} \times T_{2}\right)^{j}(x, y)\right) \hat{\omega}_{\pi(j)}(x, y)}\right)\left(\frac{\sum_{j \in B_{n}} \hat{\omega}_{\pi(j)}(x, y)}{\sum_{j \in B_{n}} \hat{\omega}_{j}(x, y)}\right) .
$$


Since $f_{1}$ is a characteristic function, by (iii) of $\alpha$-matching, this limit is between $\alpha^{2}$ and $1 / \alpha^{2}$.

Notice that we have in fact shown

$$
\alpha^{2} \leq \frac{d \nu_{z} \circ\left(I \times T_{2}\right)}{d \hat{\mu}}(x, y) \leq 1 / \alpha^{2},
$$

which completes the proof of Proposition 4.1.1.

\subsection{Controlled rank-1 maps}

From the arguments we will use in this section it is not difficult to see that there exist type III Borel measures on a topological version of Chacón's map that have minimal self-joinings. However, it is not obvious how to obtain type $\mathrm{II}_{\infty}$ examples in the context of Chacón's map. Hence we have turned to a more general context. Our examples will all be rank-1 - they are constructed by cutting and stacking with precisely one spacer sequence used.

We first briefly discuss nonsingular cutting and stacking rank-1 constructions.

The construction is as in the measure preserving case except that the measures of the columns into which the tower is subdivided will not all be equal (see e.g. [Fr], [K]). However, the Radon-Nikodym derivative of $T$ as it moves up the columns will be a constant on each level set (except for the last one), this constant being forced by the relative sizes of the intervals. (These transformations are a special case of the nonsingular transformations satisfying property $F$ introduced in [HW].)

Proposition 4.2.1. Any nonsingular rank-1 map is conservative ergodic.

Proof. We first note that the measure is clearly nonatomic and hence it suffices to show ergodicity. The same idea as in [K] for the measure preserving case applies. Suppose $A$ is a $T$-invariant set. Given $\varepsilon>0$, there is some set $L$ that is a union of levels in the $n$-block such that $\mu(A \triangle L)<\varepsilon$. Thus there is some level $L_{i}$ in $L$ such that $\mu\left(L_{i} \cap A\right)>(1-\varepsilon) \mu\left(L_{i}\right)$. But since the Radon-Nikodym derivative is constant between levels and $A$ is invariant, $\mu\left(B_{n} \cap A\right)>1-\varepsilon$, where $B_{n}$ is the $n$-block. Since $\varepsilon$ is arbitrary it follows that $A$ is the whole space $(\bmod 0)$.

We now describe a class of nonsingular rank-1 maps that we call controlled rank-1. To determine the relative proportions of the columns into which the $(n-1)$ block is cut, all that is actually necessary is that we specify the Radon-Nikodym derivative from the base of the $i$ th $(n-1)$-block in an $n$-block to the base of the $(i+1)$ st. This sequence of values determines the relative sizes of the columns into which the $(n-1)$-block must be cut to obtain these Radon-Nikodym derivatives. We always assume the Radon-Nikodym derivative from any level into the spacer is 1 .

Our spacer sequence will also have a special form. Each $(n-1)$-block in an $n$-block will be assigned a single spacer, which may be placed either below the block or above it. Let $N(n)$ be the number of columns into which we cut the $(n-1)$-block, stacking them to form the $n$-block. Hence the spacer sequence of the $N(n)(n-1)$-blocks in an $n$-block is determined by a sequence $e_{1}^{n}, \ldots, e_{N(n)}^{n}$ of 0 's 
and 1's. If $e_{i}^{n}=0$ we place the spacer of the $i$ th $(n-1)$-block before (below) the block, and if $e_{i}^{n}=1$ we place it after (above). We will assume

$$
e_{1}^{n}=1 \text { and } e_{N(n)}^{n}=0 .
$$

This ensures that no more than 2 spacers ever occur between two $n$-blocks.

To define our process all that we need give are the values $N(n)$, the sequences $\left\{e_{1}^{n}, \ldots, e_{N(n)}^{n}\right\}$, and the Radon-Nikodym derivatives $\left\{\lambda_{1}^{n}, \ldots, \lambda_{N(n)-1}^{n}\right\}$, where $\lambda_{i}^{n}$ is the Radon-Nikodym derivative from the bottom of the $i$ th $(n-1)$-block to the bottom of the $(i+1)$ st, and the form of a 0 -block. The 0 -block will be formed by stacking 3 intervals each of length $\frac{1}{3}$. As the description of the construction evolves we will place a series of restrictions on the parameters of the construction.

First, let $\left\{\varepsilon_{i}\right\}_{i \geq 1}$ be a sequence of positive values with $\sum \varepsilon_{i}<1$. We require that:

(1) For any two sub-blocks $e_{i}^{n}, e_{i+1}^{n}, \ldots, e_{i+k}^{n}$ and $e_{j}^{n}, e_{j+1}^{n}, \ldots, e_{j+k}^{n}$, where $i \neq j$ and $k \geq\left[\varepsilon_{n}^{2} N(n)\right]$,

$$
\not\left\{0 \leq t \leq k: e_{i+t}^{n}=c, e_{j+t}^{n}=d\right\}>\frac{1}{8}(k+1)
$$

for any choice of $(c, d) \in\{(0,0),(0,1),(1,0),(1,1)\}$.

(Once $\varepsilon_{n}$ is fixed, it is a standard argument (cf. [R]) that on the 2-shift, the probability of a name $e_{1}, \ldots, e_{N}$ failing to satisfy condition (1) with variable $N$ replacing $N(n)$, goes to zero exponentially in $N$. Hence if $N(n)$ is large enough we can select $\left\{e_{i}^{n}\right\}$ satisfying (1).)

(2) There is a bound $0<\gamma<1$ such that for any block $i, i+1, \ldots, i+k$, with $k<2\left[\varepsilon_{n} N(n)\right]$,

$$
\gamma \leq \prod_{j=i}^{i+k} \lambda_{j}^{n} \leq \frac{1}{\gamma} \text { and } \prod_{j=1}^{N(n)-1} \lambda_{j}^{n}=1 .
$$

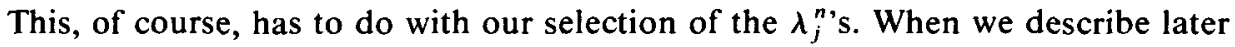
how to obtain all the various Krieger types, this will have to be obtained.

These two conditions are sufficient for our purposes. Notice the parameters can be selected inductively as follows. Having constructed $(n-1)$-blocks, we are free to select $\varepsilon_{n}$. Condition (1) places a lower bound on $N(n)$ in order to have $\left\{e_{i}^{n}\right\}$ available. Beyond this, $N(n)$ can be chosen as large as we wish, and $\left\{\lambda_{i}^{n}\right\}$ can be chosen in any way giving (2). Once $N(n)$ is fixed $\left\{e_{i}^{n}\right\}$ can be selected and the $n$-block constructed.

A construction as above governed by parameters $\left(\left\{\varepsilon_{n}\right\},\{N(n)\},\left\{e_{i}^{n}\right\},\left\{\lambda_{i}^{n}\right\}\right)$ satisfying (1) and (2) is called a controlled rank-1 map.

Suppose we have such a controlled rank-1 map. Let $B_{n}$ be the base of the $n$-block. Since $e_{1}^{n}=1, B_{n+1} \subset B_{n}$. Let $h(n)$ denote the height of the $n$-block.

$$
\begin{aligned}
h(0) & =3, \\
h(n+1) & =N(n)(h(n)+1) .
\end{aligned}
$$

Let

$$
\eta_{i}^{n}=\frac{d \mu \circ T^{i-1}}{d \mu}(x), x \in B_{n} \quad \text { and } \quad 1 \leq i \leq h(n) .
$$

Thus $\eta_{i}^{0}=1, i=1,2,3$. And inductively for $1 \leq i \leq h(n)$, 


$$
\begin{aligned}
\eta_{i}^{n+1} & =\eta_{i}^{n}, \\
\eta_{h(n)+1}^{n+1} & =\eta_{h(n)}^{n+1} .
\end{aligned}
$$

For $0<k<N(n+1)$, if $e_{k+1}^{n+1}=0$ (i.e. the spacer is below the block)

$$
\eta_{k(h(n)+1)+1}^{n+1}=\eta_{k(h(n)+1)}^{n+1}, \quad \text { (into the spacer), }
$$

and for $1 \leq i \leq h(n)$,

$$
\eta_{k(h(n)+1)+i+1}^{n+1}=\prod_{j=1}^{k} \lambda_{j}^{n+1} \eta_{i}^{n} ;
$$

if $e_{k}^{n+1}=1$ (i.e. the spacer is above the block) for $1 \leq i \leq h(n)$,

$$
\eta_{k(h(n)+1)+i+1}^{n+1}=\prod_{j=1}^{k} \lambda_{j}^{n+1} \eta_{i}^{n}
$$

and

$$
\eta_{(k+1)(h(n)+1)}^{n+1}=\eta_{k(h(n)+1)+h(n)}^{n+1}, \quad \text { (into the spacer). }
$$

We now obtain some estimates on the Radon-Nikodym derivatives of controlled rank-1 maps that will be needed in later proofs. They also given another proof of the conservativity of controlled rank-1 maps.

LEMMA 4.2.2. Let $S_{i}^{n}$ consist of all those points in the ith $(n-1)$-block in an $n$-block. If $|i-j| \leq 2\left[\varepsilon_{n} N(n)\right]$ then

$$
\gamma \leq \frac{\mu\left(S_{i}^{n}\right)}{\mu\left(S_{j}^{n}\right)} \leq \frac{1}{\gamma}
$$

Proof. For $i \leq j$,

$$
\mu\left(S_{j}^{n}\right)=\prod_{k=j}^{i-1} \lambda_{k}^{n} \mu\left(S_{i}^{n}\right) .
$$

Corollary 4.2.3. For any $1 \leq i \leq N(n)$,

$$
\mu\left(S_{i}^{n}\right) \leq \frac{1}{2 \gamma\left[\varepsilon_{n} N(n)\right]} .
$$

Proof. Let $b$ be an interval of $2\left[\varepsilon_{n} N(n)\right]$ consecutive integers in $1, \ldots, N(n)$ containing $i$. By Lemma 4.2.2,

$$
\mu\left(S_{i}^{n}\right) 2\left[\varepsilon_{n} N(n)\right] \leq \frac{1}{\gamma} \sum_{j \in b} \mu\left(S_{j}^{n}\right) \leq \frac{1}{\gamma} .
$$

COROllary 4.2.4. If b is any set of at most $100\left[\varepsilon_{n}^{2} N(n)\right]$ indices in $1, \ldots, N(n)$, then

$$
\sum_{j \in b} \mu\left(S_{j}^{n}\right) \leq \frac{100 \varepsilon_{n}}{\gamma}
$$

Proof.

$$
\frac{100\left[\varepsilon_{n}^{2} N(n)\right]}{2 \gamma\left[\varepsilon_{n} N(n)\right]} \leq \frac{100 \varepsilon_{n}^{2} N(n)}{\gamma \varepsilon_{n} N(n)} \leq \frac{100 \varepsilon_{n}}{\gamma} .
$$

(Note that (1) easily implies $\left[\varepsilon_{n}^{2} N(n)\right]>1$.) 
Corollary 4.2.5. A point $x \in X$ a.s. lies in the first or last $100\left[\varepsilon_{n}^{2} N(n)\right]$ $(n-1)$-blocks in an $n$-block for at most finitely many $n$.

LEMMA 4.2.6.

$$
\gamma \leq \frac{d \mu \circ T}{d \mu}(x) \leq \frac{1}{\gamma} .
$$

Proof. First, since

$$
\prod_{j=1}^{N(n)-1} \lambda_{j}^{n}=1, \quad \text { for } x \in B_{n}, \quad \frac{d \mu \circ T^{h(n)-1}}{d \mu}(x)=1 .
$$

Arguing inductively, the result is true for $x$ in the 0-block, except the top level. Suppose we know the result for all $x$ in $(n-1)$-blocks, except the top level. To extend the conclusion across the gaps between $(n-1)$-blocks in the $n$-block, just note that as we cross from the $i$ th to the $(i+1)$ st $(n-1)$-block, whenever we move into a spacer, $(d \mu \circ T / d \mu)(x)=1$ and when we move into the bottom level of the $(i+1)$ st block, $(d \mu \circ T / d \mu)(x)=\lambda_{i}^{n}$.

COROllary 4.2.7. For all $x$ and $n, 0 \leq k \leq 2\left[\varepsilon_{n} N(n)\right]$,

$$
\gamma^{6} \leq \frac{d \mu \circ T^{k(h(n)+1)}}{d \mu}(x) \leq \frac{1}{\gamma^{6}} .
$$

Proof. The section of orbit from $x$ to $T^{k(h(n)+1)-1}(x)$ covers a sequence of $n$-blocks. At most one gap between $n$-blocks is actually separating $m$-blocks, $m>n$, the rest are within $(n+1)$-blocks. This means that if $x$ lies at the $i$ th level of an $n$-block, then $T^{k(h(n)+1)}(x)$ lies at level $i-3, i-2, i-1, i$ or $i+1$ of an $n$-block. For any complete passage through an $n$-block we get no contribution to the Radon-Nikodym derivative. Hence the only contribution is from the gaps between $n$-blocks and the at most 3 extra terms to add or delete to complete the partial end blocks.

The contribution from the gaps is in two sequences of $\lambda_{i}^{n}$ 's of length $<2\left[\varepsilon_{n} N(n)\right]$ inside $(n+1)$-blocks and one $\lambda_{i}^{m}$.

Now use (2) and the previous lemmas to complete the proof.

Corollary 4.2.8. $\sum_{i=0}^{\infty} \omega_{i}(x)=\infty$.

Proof. In fact, for $i=h(n), \omega_{i}(x)>\gamma^{7}$.

\section{3. $\mu$-compatible measures}

In this section we continue our study of controlled rank-1 maps. All the notation introduced in $\$ 4.2$ will be used throughout. We would like to show that a controlled rank-1 map $(T, \mu)$ has minimal self-joinings over the class of all probability measures equivalent to $\mu$; we are not able to do this but in fact do show minimal self-joinings over a large enough class $\mathfrak{M}$, that we call the class of $\mu$-compatible measures. This is rich enough for our purposes (cf. Lemma 4.3.1, Theorems 3.1.11 and 3.1.12).

A measure $\mu_{i}$ equivalent to $\mu$ is said to be $\mu$-compatible if for some $\delta>0$, for all $x$ and $n$, and $0 \leq k \leq 2\left[\varepsilon_{n} N(n)\right]$,

$$
\delta \leq \frac{d \mu_{i} \circ T^{k(h(n)+1)}}{d \mu_{i}}(x) \leq \frac{1}{\delta} .
$$


LEMMA 4.3.1. (a) $\mu$ itself is $\mu$-compatible. (b) If $S$ is any $\mu$-nonsingular transformation with $S \circ T=T \circ S$, then $\mu \circ S$ is $\mu$-compatible.

Proof. (a) Let $\delta=\gamma^{5}$ and apply Corollary 4.2.7. (b) Note that

$$
\frac{d(\mu \circ S) \circ T^{j}}{d(\mu \circ S)}(x)=\frac{d \mu T^{j}}{d \mu}(S(x)) .
$$

PROPOSITION 4.3.2. If $\mu_{1}$ and $\mu_{2}$ are $\mu$-compatible and $\hat{\mu}$ is a rational joining of $\mu_{1}$ and $\mu_{2}$, then there is a $\hat{\delta}>0$ so that for $\hat{\mu}$-a.e. $(x, y)$, for all $n$ and $0 \leq k \leq 2\left[\varepsilon_{n} N(n)\right]$,

$$
\hat{\delta} \leq \frac{d \hat{\mu} \circ(T \times T)^{k(h(n)+1)}}{d \hat{\mu}}(x, y) \leq \frac{1}{\hat{\delta}} .
$$

Proof. Since $\hat{\mu}$ is rational,

$$
\frac{d \hat{\mu} \circ(T \times T)^{j}}{d \hat{\mu}}(x, y)=\frac{d \mu_{1} \circ T^{j}}{d \mu_{1}}(x) \frac{d \mu_{2} \circ T^{j}}{d \mu_{2}}(y) c_{j}(x, y),
$$

where for $\mu_{1}$-a.e. $x, c_{j}(x, y)=c_{j}(x)$ independent of $y$ for $\hat{\mu}_{x}$-a.e. $y$. Since

$$
\begin{gathered}
\int \frac{d \hat{\mu} \circ(T \times T)^{j}}{d \hat{\mu}}(x, y) d \hat{\mu}_{x}(y)=\frac{d \mu_{1} \circ T^{j}}{d \mu_{1}}(x), \text { we get } \\
c_{j}(x)=\frac{1}{\int \frac{d \mu_{2} \circ T^{j}}{d \mu_{2}}(y) d \hat{\mu}_{x}(y)},
\end{gathered}
$$

and for $j=k(h(n)+1)$,

$$
\delta \leq c_{j}(x) \leq \frac{1}{\delta}
$$

since $\mu_{2}$ is $\mu$-compatible. Thus let $\hat{\delta}=\delta^{3}$.

COROllary 4.3.3. A rational joining $\hat{\mu}$ of $\mu$-compatible measures is conservative.

We now know $\hat{\mu}$-a.e. $(x, y)$ is generic for its ergodic component $\hat{\nu}_{z}$, i.e. satisfies the Hurewicz ergodic Theorem 1.1.1.

Let $G_{j}=\left\{\left(x, T^{j}(x)\right): x \in X\right\}$ and $A=X \times X \backslash \bigcup_{j=-\infty}^{\infty} G_{j}$. These graphs are all $(T \times T)$-invariant sets.

Proposition 4.3.4. If $\hat{\mu}(A)>0$, then for $\alpha=\hat{\delta}^{2} / 16$ there is an $\alpha$-matching structure on $A$.

We develop the proof through a series of lemmas. Let $E_{N}$ be the set of all $x$ 's either between $N$-blocks, or in the first or last $100\left[\varepsilon_{n}^{2} N(n)\right](n-1)$-blocks in an $n$-block for some $n>N$.

LemmA 4.3.5. Given any $\varepsilon>0$ there is an $N_{0}$ so that for a.e. $(x, y) \in X \times X$, there is an $M(x, y)$ so that for all $n \geq N_{0}, i \leq 0 \leq j$, and $j-i+1 \geq M(x, y)$,

$$
\frac{\sum_{k=i}^{j} \chi_{E_{n}}\left(T^{k}(x)\right) \hat{\omega}_{k}(x, y)}{\sum_{k=i}^{j} \hat{\omega}_{k}(x, y)}<\varepsilon .
$$

The same holds if $x$ is replaced by $y$ in $T^{k}(x)$. 
Proof. We know $\mathbb{E}_{\hat{\mu}}\left[E_{n} \times X \mid g^{J}\right]=\mu_{1}\left(E_{n}\right)$. We know $E_{n+1} \subset E_{n}$ and by Corollary 4.2.4 $\mu_{1}\left(\bigcap_{n} E_{n}\right)=0$. Thus there is an $N_{0}$ with $\mu_{1}\left(E_{N_{0}}\right)<\varepsilon / 2$. Since $\hat{\mu}$ is conservative, for $n \geq N_{0}$ once $j-i+1$ is large enough,

$$
\varepsilon>\frac{\sum_{k=i}^{j} X_{E_{N_{0}}}\left(T^{k}(x)\right) \hat{\omega}_{k}(x, y)}{\sum_{k=i}^{j} \hat{\omega}_{k}(x, y)} \geq \frac{\sum_{k=i}^{j} X_{E_{n}}\left(T^{k}(x)\right) \hat{\omega}_{k}(x, y)}{\sum_{k=i}^{j} \hat{\omega}_{k}(x, y)}
$$

For any $x \in X$, let $1 \leq \beta_{n}(x) \leq N(n)$ be the index of the $(n-1)$-block containing $x$ in its $n$-block. If $x$ is not in an $(n-1)$-block, $\beta_{n}(x)$ is undefined. Two points $x$ and $y$ are on the same orbit if and only if $\beta_{n}(x)$ and $\beta_{n}(y)$ are asymptotically equal. By Corollary 4.2.4 we know, for a.e. $x$, once $n$ is large enough,

$$
100\left[\varepsilon_{n}^{2} N(n)\right] \leq \beta_{n}(x) \leq N(n)-100\left[\varepsilon_{n}^{2} N(n)\right] .
$$

Suppose $(x, y) \in A$, hence in distinct orbits. By the above we can find an increasing sequence $n_{i}=n_{i}(x, y)$ with $\beta_{n_{i}}(x) \neq \beta_{n_{i}}(y)$, and both lie more than $100\left[\varepsilon_{n_{i}}^{2} N\left(n_{i}\right)\right]$ from the ends of their $n_{i}$-blocks.

Consider the overlap of $n_{i}$-blocks containing $x$ and $y$ respectively. It is longer than $198\left(h\left(n_{i}-1\right)+1\right)\left[\varepsilon_{n_{i}}^{2} N\left(n_{i}\right)\right]$. Focus on a subinterval of this overlap containing the origin $a_{i} \leq 0 \leq b_{i}$ consisting of exactly $M_{i}$ full $\left(n_{i}-1\right)$-blocks with their single spacer in the $x$-orbit. Label these subintervals

$$
I_{k_{i}}, I_{k_{i}+1}, \ldots, I_{k_{i}+M_{i}-1}
$$

with $2\left[\varepsilon_{n_{i}}^{2} N\left(n_{i}\right)\right] \leq M_{i} \leq\left[\varepsilon_{n_{i}} N\left(n_{i}\right)\right]$. This interval is covered by a union of complete $\left(n_{i}-1\right)$-blocks, with their spacers, in the $y$-orbit

$$
J_{k_{i}^{\prime}-1}, J_{k_{i}^{\prime}}, \ldots, J_{k_{i}^{\prime}+M_{i}-1} \text {. }
$$

The subscripts $t$ of $I_{t}, J_{t}$ are the indices of the $\left(n_{i}-1\right)$-blocks in their $n$-blocks. Thus

$$
I_{j} \subset J_{k_{i}^{\prime}-k_{i}+j-1} \cup J_{k_{i}^{\prime}-k_{i}+j}=J_{j+d_{i}-1} \cup J_{j+d_{i}} .
$$

We say that $i$ is a bad index if either $d_{i}$ or $d_{i}-1=0$ and

$$
\#\left(I_{j} \cap J_{j}\right)>100\left[\varepsilon_{n_{i}}^{2} N\left(n_{i}-1\right)\right]\left(h\left(n_{i}-2\right)+1\right) .
$$

The reason this is 'bad' is that the overlaps of $\left(n_{i}-1\right)$-blocks is substantial on indices which agree.

LEMMA 4.3.6. If $i$ is a bad index, then $i+1$ is not.

Proof. If $i$ is bad then the $n_{i}$-blocks containing $x$ and $y$ overlap in at least $h\left(n_{i}\right)-h\left(n_{i}-1\right)$ places. But then for any $n, n_{i+1}>n>n_{i}$, the $n$-blocks containing $x$ and $y$ overlap in at least $h(n)-h\left(n_{i}-1\right)$ places, as $\beta_{n}(x)=\beta_{n}(y)$. Thus in the $n_{i+1}$-block when we consider $\left[a_{i+1}, b_{i+1}\right]$, the overlaps of $\left(n_{i+1}-1\right)$-blocks are either of distinct indices or of length at most

$$
h\left(n_{i}-1\right) \leq h\left(n_{i+1}-2\right)<100\left[\varepsilon_{n_{i+1}-1}^{2} N\left(n_{i+1}-1\right)\right]\left(h\left(n_{i+1}-2\right)+1\right) .
$$

We drop to a subsequence and assume no $i$ is bad. Thus we always have one of two cases:

(i) both $d_{i}$ and $d_{i}-1$ are nonzero, or 
(ii) if one is zero, it corresponds to an overlap shorter than $100\left[\varepsilon_{n_{i-1}}^{2} N\left(n_{i}-i\right)\right] \times$ $\left(h\left(n_{i}-2\right)+1\right)$, i.e. in the first or last $100\left[\varepsilon_{n_{i}-1}^{2} N\left(n_{i}-1\right)\right]$ of the $\left(n_{i}-2\right)$-blocks in the $\left(n_{i}-1\right)$-block.

By Lemma 4.3.5 such indices contribute little once $i$ is large enough.

In case (ii) above one of the two $J_{j+d_{i}-1}, J_{j+d_{i}}$ has already been identified as 'larger', the longer one. We call it $J_{j+\tilde{d}_{i}}$. In case (i) we also want to make a selection of a 'larger' one. Consider which of the two sums

$$
\sum_{i \in I_{h_{i}} \cap J_{h_{i}+d_{i}-1}} \hat{\omega}_{i}(x, y), \quad \sum_{i \in I_{h_{i}} \cap J_{h_{i}+d_{i}}} \hat{\omega}_{i}(x, y)
$$

is larger. Let $\bar{d}_{i}$ be such that the sum over $I_{k_{i}} \cap I_{k_{i}+\bar{d}_{i}}$ is the larger one. Notice $\bar{d}_{i} \neq 0$.

LEMMA 4.3.7. If $i$ is large enough, for any $k_{i} \leq j \leq k_{i}+M_{i}-1$,

$$
\sum_{i \in I_{j} \cap J_{i+d_{i}}} \hat{\omega}_{i}(x, y) \geq \frac{\hat{\delta}^{2}}{2 M_{i}} \sum_{i=a_{i}}^{b_{i}} \hat{\omega}_{i}(x, y) .
$$

Proof. Now $\left[a_{i}, b_{i}\right]=\bigcup_{k=0}^{M_{i}-1} I_{k_{i}}+k\left(h\left(n_{i}-1\right)+1\right)$, so for any $k_{i} \leq j \leq k_{i}+M_{i}-1$,

$$
\left.\sum_{i=a_{i}}^{b_{i}} \hat{\omega}_{i}(x, y)=\sum_{k=k_{i}-j}^{k+M_{i}-1-j} \sum_{t \in I_{i}} \hat{\omega}_{t}(x, y) \hat{\omega}_{k\left(h\left(n_{i}-1\right)+1\right)}\right)\left(T^{t}(x), T^{\prime}(y)\right) .
$$

By Proposition 4.3.2 then

$$
\sum_{t \in I_{i}} \hat{\omega}_{r}(x, y) \geq \frac{\hat{\delta}}{M_{i}} \sum_{t=a_{i}}^{b_{i}} \hat{\omega}_{t}(x, y) .
$$

We also know from Proposition 4.3.2 that

$$
\hat{\delta} \leq \sum_{t \in I_{i} \cap J_{i+\bar{d}_{i}}} \hat{\omega}_{t}(x, y) / \sum_{t \in I_{h_{i}} \cap J_{k_{i}+\bar{d}_{i}}} \hat{\omega}_{i}(x, y) \leq \frac{1}{\hat{\delta}} .
$$

In case (i) we can conclude, for all $k_{i} \leq j \leq k_{i}+M_{i}-1$,

$$
\sum_{i \in I_{i} \cap J_{i+\bar{d}_{i}}} \hat{\omega}_{i}(x, y) \geq \frac{\hat{\delta}^{2}}{2\left(M_{i}\right)} \sum_{j=a_{i}}^{b_{i}} \hat{\omega}_{j}(x, y) .
$$

In case (ii), by Lemma 4.3 .5 , for any $\varepsilon$ once $i$ is large enough

$$
\frac{\sum_{j=k_{i}}^{k_{i}+M_{i}-1} \sum_{t \in I_{i} \cap J_{i+\bar{c}_{i}}} \hat{\omega}_{t}(x, y)}{\sum_{j=a_{i}}^{b_{i}} \hat{\omega}_{j}(x, y)} \geq 1-\varepsilon
$$

as the upper sum is over indices $t$ with $T^{t}(x) \in E_{n-1}^{c}$.

Make $(1-\varepsilon)>\delta$ and for some $j_{0}$ we must have

$$
\sum_{i \in I_{i_{1}} \cap J_{i_{0}+d_{i}}} \hat{\omega}_{i}(x, y) \geq \frac{\hat{\delta}}{M_{i}} \sum_{j=a_{i}}^{b_{i}} \hat{\omega}_{j}(x, y) .
$$

Replacing $k_{i}$ by $j_{0}$ in expression (4.3.1) gives case (ii).

The sets $B_{n}$ and $B_{n}^{\prime}$ will be unions of sets $I_{j} \cap J_{j+\bar{e}_{i}}$. Since $M_{i} \geq 2\left[\varepsilon_{n_{i}}^{2} N\left(n_{i}\right)\right]$ and $\bar{d}_{i} \neq 0$ we know that among the pairs $\left(e_{i}^{n_{i}}, e_{j+\bar{d}_{i}}^{n_{j}}\right), k_{i} \leq j \leq k_{i}+M_{i}-1$, we see a fraction of at least $\frac{1}{8}$ of each possibility, $(0,0),(0,1),(1,0)$ and $(1,1)$. Thus we can find two 
subsets $\Lambda_{i}, \Lambda_{i}^{\prime} \subset\left[k_{i}, k_{i}+M_{i}-1\right]$ with the same size

$$
\#\left[\Lambda_{i}\right]=\#\left[\Lambda_{i}^{\prime}\right] \geq\left(\frac{1}{8}\right) M_{i},
$$

and for $j \in \Lambda_{i},\left(e_{j}^{n_{1}}, e_{j+\bar{d}_{i}}^{n_{1}}\right)=(0,0)$, i.e. both spacers come before the block, and for $j \in \Lambda_{i}^{\prime},\left(e_{j}^{n_{i}}, e_{j+\bar{d}_{i}}^{n_{i}}\right)=(0,1)$, i.e. the $x$-spacer is before, the $y$-spacer is after the block.

Let $\bar{\pi}$ be any bijection of $\Lambda_{i}$ to $\Lambda_{i}^{\prime}$. Set

$$
B_{i}=\bigcup_{j \in \Lambda_{i}} I_{j} \cap J_{j+\bar{d}_{i}} \quad \text { and } \quad B_{i}^{\prime}=\bigcup_{j \in \Lambda_{i}^{\prime}} I_{j} \cap J_{j+\bar{d}_{i}}
$$

Define $\pi_{i}: B_{i} \rightarrow B_{i}^{\prime}$ by sending $I_{j} \cap J_{j+\bar{d}_{i}}$ to $I_{\bar{\pi}(j)} \cap J_{\bar{\pi}(j)+\bar{d}_{i}}$ by translation by $(\bar{\pi}(j)-j) \times$ $\left(h\left(n_{i}-1\right)+1\right)$.

Let $P_{i}$ be the partition of $X$ into the levels of the $(i-1)$ st tower and its complement. Thus $\left\{P_{i}\right\}$ is a refining and generating sequence of partitions.

LEMMA 4.3.8.

(a) Both $B_{i}$ and $B_{i}^{\prime}$ are disjoint unions of intervals, all of the same length, and this length goes to infinity with $i$.

(b) $\sum_{j \in B_{i}} \hat{\omega}_{j}(x, y) \geq \frac{\hat{\delta}^{2}}{16} \sum_{j=a_{i}}^{b_{i}} \hat{\omega}_{i}(x, y)$

and

$$
\sum_{j \in B_{i}^{\prime}} \hat{\omega}_{j}(x, y) \geq \frac{\hat{\delta}^{2}}{16} \sum_{j=a_{i}}^{b_{i}} \hat{\omega}_{j}(x, y),
$$

(c) for $j \in B_{i}$,

$$
\hat{\delta} \geq \frac{\hat{\omega}_{j}(x, y)}{\hat{\omega}_{\pi_{i}(j)}(x, y)} \geq \frac{1}{\delta},
$$

(d) for $j \in B_{n}$,

$$
\begin{aligned}
& T^{j}(x) \in P_{i, l} \text { iff } T^{\pi_{i}(j)}(x) \in P_{i, l}, \quad \text { and } \\
& T^{j}(y) \in P_{i, l} \text { iff } T^{\pi_{i}(j)-1}(y) \in P_{i, l} .
\end{aligned}
$$

Proof. We know all the $I_{j} \cap J_{j+\bar{d}_{i}}$ are of the same length. As we chose $\bar{d}_{i}$ to give us the 'larger' sum, by Lemma 4.3.5 this length could not stay bounded on any subsequence.

For (b) we just note that $B_{i}, B_{i}^{\prime}$ contain at least $\frac{1}{8}$ in cardinality of the $I_{j} \cap J_{j+\bar{d}_{i}}$ and apply Lemma 4.3.7.

For (c) note $\pi(j)-j=k(h(n)+1)$ with $|k| \leq\left[\varepsilon_{n_{i}} N\left(n_{i}\right)\right]$, so we obtain the bounds from Proposition 4.3.2.

For (d), of course, just note that $(i-1) \leq n_{i}-1$ and the effect of moving the spacer in the $y$-block from below to above shifts $\pi_{i}(y)$ one level higher in the tower.

Proof of Proposition 4.3.4. Let $\alpha=\hat{\delta}^{2} / 16$. Now just drop to a subsequence of $n_{i}$ 's to give the precise bound in (i) of $\alpha$-matching. 
Corollary 4.3.9. $T \times T$ is ergodic, hence weak mixing (cf. [ALW]).

Proof. For $\hat{\mu}=\mu \times \mu, A=X \times X$ and hence a.e.- $\nu_{z}$ is $I \times T$ invariant and hence equals $\mu \times \mu$.

TheOREM 4.3.10. Let $(X, \mu, T)$ be a controlled rank-1 map. Let $\mathfrak{M}$ be the class of $\mu$-compatible measures. Then $T$ has minimal self-joinings over $\mathfrak{M}$.

Proof. Let $\hat{\mu}$ be a rational joining. First write $X \times X=\bigcup_{j=-\infty}^{\infty} G_{j} \cup A$, where $G_{j}=$ $\left\{\left(x, T^{j}(x)\right): x \in X\right\}$ and $A$ is the remainder. We have seen by Propositions 4.1.1 and 4.3.4 that for any ergodic component $\nu_{z}, z \subset A, \nu_{z}$ is $I \times T$-nonsingular. By Proposition 3.1.5, $\nu_{2}=\mu_{1} \times \mu_{2}$ and we can write

$$
\hat{\mu}=\hat{\mu}(A) \mu_{1} \times \mu_{2}+\hat{\mu}\left(A^{c}\right) \hat{\mu}_{0}, \quad \text { where } \hat{\mu}_{0} \text { is graphic. }
$$

Question $D$. Are there measures $\mu_{1}$ and $\mu_{2}$ equivalent to $\mu$ but not $\mu$-compatible for which $T$ fails to have minimal self-joinings? More generally, if $T$ has minimal self-joinings with respect to some measure equivalent to $\mu$, must it have minimal self-joinings with respect to all? If not, are there examples of systems with minimal self-joinings with respect to all measures equivalent to $\mu$ ?

COROLlary 4.3.11. Any controlled rank-1 map has no nontrivial factor algebras and the only nonsingular maps it commutes with are its powers.

Now we are ready to show how to obtain controlled rank-1 maps for each Krieger class $\mathrm{II}_{1}, \mathrm{II}_{\infty}$, and $\mathrm{III}_{\lambda}, 0 \leq \lambda \leq 1$.

\subsection{Type $I I_{1}$ example}

This is almost trivial. Simply set all $\lambda_{i}^{n}=1$. This gives a finite measure preserving transformation that has minimal self-joinings in the usual sense.

\subsection{Type $I_{\infty}$ example}

We choose $\varepsilon_{n}=1 / n 20^{n}$. Now condition (1) gives a lower limit for $N(n)$. Split the block of $N(n)(n-1)$-blocks into five sections. The first, third and fifth of length $N(n)\left(1-4 n \varepsilon_{n}\right) / 3$, and the second and fourth, which connect them, of length $2 n \varepsilon_{n} N(n)$.

For $i$ in the first, third or fifth section $\lambda_{i}^{n}=1$. For $i$ in the second, $\lambda_{i}^{n}=\left(\frac{1}{20}\right)^{1 / 2 \varepsilon_{n} N(n)}$, and for $i$ in the fourth section, $\lambda_{i}^{n}=(20)^{1 / 2 \varepsilon_{n} N(n)}$. Thus the product of the $\lambda_{i}^{n}$ 's across sections 2,3 and 4 is 1 .

For any $k<2\left[\varepsilon_{n} N(n)\right]$ we get

$$
\frac{1}{20} \leq \prod_{i=j}^{j+k} \lambda_{i}^{n} \leq 20
$$

Thus we have a controlled rank-1 map. Let $X_{n}$ be those points in the levels of the $n$-block corresponding to sections 1 and 5 . Now since

$$
\prod_{i \in \text { section } 2} \lambda_{i}^{n}=\left(\frac{1}{20}\right)^{n}
$$

section 3 has measure $\left(\frac{1}{20}\right)^{n}$ times that of sections 1 or 5 . Sections 2 and 4 each have 
the same measure, at most $12 n \varepsilon_{n}$ times that of section 1 or 5 . Thus

$$
\mu\left(X_{n}^{c}\right) \leq \frac{13}{20^{n}} \mu\left(X_{n}\right)<\frac{13}{20^{n}} .
$$

Now since $\sum_{n} \mu\left(X_{n}^{c}\right)<1, \mu\left(\bigcap_{n \geq 1} X_{n}\right)>0$. It is an easy induction that if $x, T^{j}(x) \in$ $\bigcap_{n \geq 1} X_{n}$ then $\left(d \mu \circ T^{j} / d \mu\right)(x)=1$. Thus setting $Y=\bigcap_{n \geq 1} X_{n}, T$ induced on $Y$ is measure preserving, hence $T$ itself is either type $\mathrm{II}_{1}$ or $\mathrm{II}_{\infty}$.

Notice that on an orbit, occurrences of $X_{n}$ come in blocks of length

$$
\frac{N(n)\left(1-4 n \varepsilon_{n}\right)}{3}(h(n-1)+1)
$$

alternately separated by a long block of length

$$
\frac{N(n)+4 n \varepsilon_{n}}{3}(h(n-1)+1)
$$

and a short block of length 0,1 or 2 . Letting $Y_{n}=\bigcap_{i=1}^{n} X_{i}$, it easily follows that

$$
\limsup _{N \rightarrow \infty} \frac{1}{N} \sum_{i=0}^{N-1} \chi_{Y_{n}}\left(T^{i}(x)\right) \leq \frac{1}{3^{n}}
$$

Hence

$$
\lim _{N \rightarrow \infty} \frac{1}{N} \sum_{i=0}^{N-1} \chi_{Y}\left(T^{i}(x)\right)=0 \text { a.e. }
$$

and $(T, \mu)$ cannot be of type $I_{1}$.

As discussed in the introduction, Aaronson and Nadkarni in [AN] construct an example of an infinite measure preserving group rotation that has no nontrivial $\sigma$-finite invariant sub- $\sigma$-algebras; however their example has $0-\infty$ invariant sub- $\sigma$ algebras. Maharam has asked, independently of $[\mathbf{A}]$, whether there exist infinite measure preserving transformations with no $0-\infty$ invariant sub- $\sigma$-algebras; our example clearly satisfies this.

4.6. Type $I I I_{\lambda}$ example $(0<\lambda<1$.)

Let $N(n)$ be odd for all $n$ and set

$$
\begin{aligned}
& \lambda_{i}^{n}=\lambda, \text { for } i \text { odd, } \\
& \lambda_{i}^{n}=\frac{1}{\lambda}, \text { for } i \text { even. }
\end{aligned}
$$

It is easy to check that we have a controlled rank-1 map with $\gamma=\lambda$. The ratio set of $T$ is obviously contained in the powers of $\lambda$. We wish to show $\lambda$ is in the ratio set.

Let $A \subset X, \mu(A)>0$. Select $n$ large enough so that for some level set $L^{\prime}$ in the n-block,

$$
\mu\left(L^{\prime} \cap A\right) \geq\left(1-\frac{\lambda}{1000}\right) \mu\left(L^{\prime}\right) .
$$

In the $(n+1)$-block $L^{\prime}$ is the union of one level from each occurrence of the $n$-block. 
Write $L=L_{1} \cup L_{2}$, where $L_{1}$ consists of those occurrences in odd index $i<N(n)$ and $L_{2}$ those in even index $n$-blocks. For any $x \in L_{1}$ there is a unique

$$
j(x) \in\{h(n), h(n)+1, h(n)+2\}, \quad \text { with } T^{j(x)}(x) \in L_{2} .
$$

The choice depends on whether there are 0,1 or 2 spacers between the $n$-blocks. Furthermore, $\left(d \mu \circ T^{j(x)} / d \mu\right)(x)=\lambda$ and the map $g: x \rightarrow T^{j(x)}(x)$ is a bijection of $L_{1}$ to $L_{2}$. Thus $\mu\left(L_{2}\right)=\lambda \mu\left(L_{1}\right)$. Since

$$
\mu(L \cap A) \geq\left(1-\frac{\lambda}{100}\right) \mu(L)
$$

we get

$$
\mu\left(L_{i} \cap A\right) \geq 0.98 \mu\left(L_{i}\right), \quad \text { for } i=1,2
$$

Thus

$$
\begin{aligned}
\mu\left(g\left(L_{1} \cap A\right)\right) & \geq 0.98 \mu\left(L_{2}\right), \quad \text { and } \\
\mu\left(L_{2} \cap A\right) & \geq 0.98 \mu\left(L_{2}\right) .
\end{aligned}
$$

Thus

$$
\mu\left(g\left(L_{1} \cap A\right) \cap\left(L_{2} \cap A\right)\right)>0 .
$$

As $j(x)$ takes on only three values, we can select a subset $A_{1} \subset L_{1} \cap A$, $\mu\left(A_{1}\right) \geq\left(\frac{1}{3}\right) \mu\left(L_{1} \cap A\right)>0$, and on $A_{1}, j(x)=j$ is a constant. Thus $A_{1} \subset A$, $T^{j}\left(A_{1}\right) \subset A$ and $\left(d \mu \circ T^{j(x)} / d \mu\right)(x)=\lambda$ for all $x \in A_{1}$. Therefore $T$ is type III $_{\lambda}$.

4.7. Type $I I I_{1}$ example

To get the ratio set to be all of $\mathbb{R}^{+} \cup\{0\}$ select $0<\lambda_{1}, \lambda_{2}<1$ with $\log \left(\lambda_{1}\right)$ and $\log \left(\lambda_{2}\right)$ irrationally related. Do the construction as in Example 4.6 except that when building $n$-blocks, for $n$ odd use $\lambda_{1}$, and for $n$ even use $\lambda_{2}$. Repeating the above argument both $\lambda_{1}, \lambda_{2}$ are in the ratio set. As the ratio set $(\backslash\{0\})$ is a closed multiplicative subgroup it is all of $\mathbb{R}^{+} \cup\{0\}$.

4.8. Type $I I_{0}$ example

This construction is similar to that of example 4.5, using five sections. Define $\gamma(n)$ inductively by $\gamma(1)=1, \gamma(n+1)=(n+1) \prod_{i=1}^{n} \gamma(i)$.

Let $\varepsilon_{n}=1 / \log _{2}(\gamma(n)) 2^{\gamma(n)}$. Condition (1) sets a lower bound on $N(n)$. Let $g(n)=N(n) / 2^{\gamma(n)}$. We assume $2^{\gamma(n)}$ divides $N(n)$. Let

$$
k(n)=\frac{N(n)-2 g(n)}{2+\gamma(n)}=N(n)\left(\frac{2^{\gamma(n)}-2}{(2+\gamma(n)) 2^{\gamma(n)}}\right),
$$

which we also assume is an integer. Thus $g(n)=\varepsilon_{n} \log _{2}(\gamma(n)) N(n)$ and

$$
N(n)=(2+\gamma(n)) k(n)+2 g(n) .
$$

The five sections will have lengths $k(n), g(n), \gamma(n) k(n), g(n)$ and $k(n)$ in that order. As in Example 4.5, across sections 1,3 and $5, \lambda_{i}^{n}=1$. Across section 2,

$$
\begin{aligned}
& \lambda_{i}^{n}=\left(\frac{1}{2}\right)^{1 / \varepsilon_{n} N(n)}, \text { and across section } 4 \\
& \lambda_{i}^{n}=(2)^{1 / \varepsilon_{n} N(n)} .
\end{aligned}
$$


Thus for any $0 \leq k \leq \varepsilon_{n} N(n)$,

$$
\frac{1}{2} \leq \prod_{i=j}^{j+k} \lambda_{i}^{n} \leq 2, \quad \prod_{i=1}^{N(n)-1} \lambda_{i}^{n}=1,
$$

and we have a controlled rank-1 map.

Also notice

$$
\begin{aligned}
\mu(\text { section } 3) & =\gamma(n) 2^{-\log _{2}(\gamma(n))} \mu(\text { section } 1) \\
& =\mu(\text { section } 1)=\mu(\text { section } 5) .
\end{aligned}
$$

Further,

$$
\mu(\text { section } 2)=\mu(\text { section } 4) \leq \frac{g(n)}{k(n)} \mu(\text { section } 1)<\frac{1}{2^{\gamma(n)}} .
$$

In other words, sections 2 and 4 have negligible mass and sections 1, 3 and 5 have equal mass, but 3 is $n$-times as long as 1 and 5 .

We first show only 0 and 1 can be in the ratio set. Let $E_{n}$ be the set of all points in the second and fourth sections of the $n$-block.

$$
\mu\left(\bigcup_{i=3}^{\infty} E_{n}\right) \leq 2 \sum_{i=3}^{\infty} \frac{1}{2^{\gamma(n)}}<\frac{1}{2} .
$$

Set $E=\bigcup_{i=3}^{\infty} E_{n}$. Pick $\frac{1}{3}>\varepsilon>0$ and now choose $n$ with $1 / \gamma(n)<\varepsilon$ and $L$ a level set in the $n$-block with $\mu(L \cap E)>0$. If $x \in L \cap E$ and for some $j>0, T^{j}(x) \in L \cap E$, then $j$ moves us from an odd section to an odd section of some $m$-block, $m>n>3$. In this case

$$
\frac{d \mu \circ T^{j(x)}}{d \mu}(x)=1 \text { or is }>\frac{\gamma(m)}{\prod_{i=1}^{m-1} \gamma(i)}>m \text { or is }<\frac{\prod_{i=1}^{m-1} \gamma(i)}{\gamma(m)}<1 / m,
$$

hence $=1$ or is $<\varepsilon$ or $>1 / \varepsilon$.

To see that 0 is in the ratio set, notice first that if $L$ is a level of an $n$-block, then for all $m>n,\left(\frac{1}{3}-1 / 2^{n-2}\right) \mu(L)$ lies in each of sections 1,3 and 5 of the $m$-block.

For any set $A, \mu(A)>0$, and $\varepsilon>0$, select $n$ with $1 / \gamma(n)<\varepsilon,\left(\frac{1}{3}-1 / 2^{n-2}\right)>\frac{1}{10}$ and so that some level set $L$ of the $n$-block has $\mu(A \cap L)>0.99 \mu(L)$. Thus in the $(n+1)$-block there must be level sets $L_{1}, L_{3}$ each in sections 1 and 3 respectively, all contained in $L$, with $\mu\left(A \cap L_{i}\right)>0.98 \mu\left(L_{i}\right)$. Now $L_{3}=T^{j}\left(L_{1}\right)$ and for $x \in L_{2}$,

$$
\frac{d \mu \circ T^{j(x)}}{d \mu}(x)=\frac{1}{\gamma(n+1)}
$$

a constant. Thus

$$
\mu\left(T^{j}\left(A \cap L_{1} \cap\left(A \cap L_{3}\right)\right) \geq 0.96 \mu\left(L_{3}\right) .\right.
$$

Letting $A_{1}=A \cap L_{1} \cap T^{-j}(A), \mu\left(A_{1}\right)>0, A_{1} \subset A$, and for $x \in A_{1}$,

$$
\frac{d \mu \circ T^{j(x)}}{d \mu}(x)=\frac{1}{\gamma(n+1)}<\varepsilon .
$$




\section{REFERENCES}

[A] J. Aaronson. The intrinsic normalising constants of transformations preserving infinite measures. J. d'Analyse Math. 49 (1987), 239-270.

[ALW] J. Aaronson, M. Lin \& B. Weiss. Mixing properties of Markov operators and ergodic transformations, and ergodicity of Cartesian products. Israel J. Math. 33 (1979), 198-224.

[AN] J. Aaronson \& M. Nadkarni, $L_{\infty}$ eigenvalues and $L_{2}$ spectra of non-singular transformations. Proc. London Math. Soc. 55 (3) (1987), 538-570.

[EHI] S. J. Eigen, A. Hajian \& Y. Ito. Ergodic measure preserving transformations of finite type. Tokyo J. Math. 11 (2) 1988, 459-470.

[Fr] N. A. Friedman. Introduction to Ergodic Theory. Van Nostrand: New York, 1970.

[F1] H. Furstenberg. Disjointness in ergodic theory, minimal sets, and a problem in Diophantine approximation. Math. Systems Theory 1 (1967), 1-49.

[F2] H. Furstenberg. Recurrence in Ergodic Theory and Combinatorial Number Theory. Princeton University Press: Princeton, 1981.

[HK] A. Hajian \& S. Kakutani. Example of an ergodic measure preserving transformation on an infinite measure space. Contributions to Ergodic Theory and Probability. Lecture Notes in Mathematics 160. Springer-Verlag: Berlin, 1970, 45-52.

[HIK] A. Hajian, Y. Ito \& S. Kakutani. Invariant Measures and orbits of dissipative transformations. Adv. Math. 9 (1972), 52-65.

[H] P. R. Halmos. An ergodic theorem. Proc. Nat. Acad. Sci. U.S.A. 32 (1946), 156-161.

[HO] T. Hamachi \& Osikawa. Ergodic groups of automorphisms and Krieger's theorems. Seminar on Math. Sci. Keio University No. 3 (1981).

[HW] J. Hawkins \& E. J. Woods. Approximately transitive diffeomorphisms of the circle. Proc. Amer. Math. Soc. 90 (1984), 252-262.

[JK] L. K. Jones \& U. Krengel. On transformations without finite invariant measure. Adv. Math. 12 (1974), 275-295.

[JRS] A. del Junco, M. Rahe \& L. Swanson. Chacón's automorphism has minimal self-joinings. J. d'Analyse Math. 37 (1980), 276-284.

[JR] A. del Junco \& D. J. Rudolph. On ergodic actions whose self-joinings are graphs. Ergod. Th. \& Dynam. Sys. 7 (1987), 531-557.

[K] J. King. Joining-rank and the structure of finite rank mixing transformations. J. d' Analyse Math. 51 (1988), 182-227.

[Kr] W. Krieger. On the Araki-Woods asymptotic ratio set and non singular transformations of a measure space. Lecture Notes in Mathematics 160. Springer-Verlag: Berlin, 1970, 158-177.

[M1] D. Maharam. Incompressible transformations. Fund. Math. LVI (1964), 35-50.

[M2] D. Maharam. On the planar representation of a measurable subfield. Measure Theory Oberwolfach 1983. Lecture Notes in Mathematics 1089. Springer-Verlag: Berlin, 1984, 47-57.

[P] W. Parry. Topics in Ergodic Theory. Cambridge University Press: Cambridge, 1981.

[R] D. J. Rudolph. An example of a measure preserving map with minimal self-joinings, and applications. J. d'Analyse Math. 35 (1979), 97-122.

[S] K. Schmidt. Lectures on cocycles of ergodic transformation groups. Macmillan Lectures in Math. 1. Macmillan Co. of India: New Delhi, 1977.

[W] B. Weiss. Orbit equivalence of nonsingular actions. Monographie No. 29. L'Enseignement Mathématique (1980), 77-107. 